\title{
Performance Analysis of AOA-Based Localization Using the LS Approach: Explicit Expression of Mean-Squared Error
}

\author{
Byung-Kwon Son, Do-Jin An, and Joon-Ho Lee \\ Department of Information \& Communication Engineering, Sejong University, Seoul, Republic of Korea \\ Correspondence should be addressed to Joon-Ho Lee; joonhlee@sejong.ac.kr
}

Received 11 December 2019; Accepted 14 May 2020; Published 18 August 2020

Academic Editor: Vincenzo Stornelli

Copyright (c) 2020 Byung-Kwon Son et al. This is an open access article distributed under the Creative Commons Attribution License, which permits unrestricted use, distribution, and reproduction in any medium, provided the original work is properly cited.

\begin{abstract}
In this paper, a passive localization of the emitter using noisy angle-of-arrival (AOA) measurements, called Brown DWLS (Distance Weighted Least Squares) algorithm, is considered. The accuracy of AOA-based localization is quantified by the mean-squared error. Various estimates of the AOA-localization algorithm have been derived (Doğançay and Hmam, 2008). Explicit expression of the location estimate of the previous study is used to get an analytic expression of the mean-squared error (MSE) of one of the various estimates. To validate the derived expression, we compare the MSE from the Monte Carlo simulation with the analytically derived MSE.
\end{abstract}

\section{Introduction}

There has been a great deal of research on the determination of emitter location. Localization consists of two parts: measuring localization parameters between nodes and with these parameters to estimate location. The localization parameters can be AOA and TOA (time of arrival). AOA-based localization schemes have been considered [1-9]. The AOA-based localization algorithm can be classified as follows: linear least-squared (LS) estimation [10], nonlinear least-squared estimation [11,12], minimum mean-squared error (MMSE) estimation with Kalman filter [13], and maximum likelihood (ML) estimation [14].

In [10], least-squared (LS) algorithm for emitter localization is proposed. In the algorithm, the distances between the given bearing lines to emitter location estimate are calculated as a function of emitter location estimate. Cost function is defined from the sum of squares of the distances, and the location estimate is obtained from the location minimizing the cost function. In $[11,12]$, nonlinear least-squared algorithm is used for the emitter localization. In [13], since the bearing angle measurements are noisy, the measurements are combined using a nonlinear least squares filter or an extended Kalman filter to obtain the optimal filtered position estimate. The maximum likelihood (ML) and the Stansfield algorithm for
AOA-based localization have been considered in [14], where performances in terms of the bias and the covariance matrix for these AOA-based localization algorithms have been presented analytically. In the proposed scheme, least-squared (LS) algorithm is employed to get location estimate using noisy AOA measurements. Explicit expressions of localization error and the mean-squared error (MSE) are derived. There is also a technique called total least-squared (TLS) estimation [15], which is an extension of the LS estimation.

In this paper, we are concerned with estimating the location of the single stationary target by using the received signals at the moving sensor. We assume that the locations of the moving sensor are available. It is assumed that these is no uncertainty in sensor location. Also, it is assumed that the speed of the moving sensor is constant, which implies, given the trajectory of the moving sensor, every location of the moving sensor at the instants, when the LOB measurement is given, can be specified by the LOB measurement interval.

Note that, in the LS-based linear bearing-based localization algorithm, the speed of the moving sensor is not necessarily constant. The assumption of constant moving speed is just for convenience in implementation of the algorithm in the numerical results. Gaussian random variable representing LOB measurement error at each sensor location is adopted. Means of these Gaussian random variables are set 
to be identically zero. Generally, it can be regarded as a thermal noise component generated inside the receiver. By exploiting this observation, we try to get an analytic expression of the location estimate and the analytic expression of the MSE of the location estimate.

AOA estimation errors as well as sensor location errors are responsible for the errors in the location estimate. In [16], it is assumed that sensor locations are available without uncertainty and that the AOA estimation errors can be modelled as Gaussian random variables. Explicit expression of the location estimate in these assumptions has been derived in [16]. In this paper, using the results presented in [16], an explicit expression of the MSE of the location estimate is derived. In [17], an iterative solution based on ML approach has been presented and its performance has been illustrated.

In this paper, we present more explicit expression of the MSE. Our expression is explicit and intuitive in that the estimation error and the MSE are expressed in terms of the AOA estimation error. Therefore, the contribution of this paper is to present an analytic expression of the MSE of the LS-based localization algorithm. In this paper, two approximations named $K$-approximation and $L$-approximation have been employed to derive the mean-squared error (MSE) of location estimate.

Taylor series expansion has been used in $K$-approximation. $K$-approximation has been adopted to get polynomial approximations of various sinusoids. Many studies have been conducted on error analysis due to various nonlinear approximations $[18,19]$.

\section{LS-Based Location Estimate [10]}

Let $\left(x_{i}, y_{i}\right)$ and $\phi_{i}$ denote the $i$ th sensor location and the AOA measurement at $\left(x_{i}, y_{i}\right)$. Given $\mathbf{x}=\left(x_{i}, y_{i}\right)^{T}$ and $\phi_{i}$ for $1 \leq i \leq$ $N$, where $N$ is the number of sensor coordinates, we are to estimate $\mathbf{x}_{T}=\left(x_{T}, y_{T}\right)$, which denotes the true emitter location. $\mathbf{x}$ estimation of the emitter location can be written as [10]

$$
\mathbf{A x}=\mathbf{b}
$$

where

$$
\begin{gathered}
\mathbf{A}=\left[\begin{array}{cc}
\sin \phi_{1} & -\cos \phi_{1} \\
\sin \phi_{2} & -\cos \phi_{2} \\
\vdots & \vdots \\
\sin \phi_{N} & -\cos \phi_{N}
\end{array}\right] \\
\mathbf{b}=\left[\begin{array}{c}
x_{1} \sin \phi_{1}-y_{1} \cos \phi_{1} \\
x_{2} \sin \phi_{2}-y_{2} \cos \phi_{2} \\
\vdots \\
x_{N} \sin \phi_{N}-y_{N} \cos \phi_{N}
\end{array}\right] .
\end{gathered}
$$

The linear least-squared (LS) estimation algorithm for estimating the emitter location is briefly described. Since $\mathbf{A}$ is not invertible, the following normal equation is obtained from (1) for the LS estimate:

$$
\left(\mathbf{A}^{H} \mathbf{A}\right) x=\mathbf{A}^{H} \mathbf{b} .
$$

The location estimate for the noiseless AOA measurements can be written as

$$
\mathbf{x}=\left(\mathbf{A}^{H} \mathbf{A}\right)^{-1} \mathbf{A}^{H} \mathbf{b}
$$

Noiseless AOA is denoted by $\phi_{i}$ and noisy AOA is denoted $\phi_{i}+\delta \phi_{i}$, where $\delta \phi_{i}$ denotes an error in AOA measurement. Under these conditions, $\mathbf{x}^{\prime}$ denotes an estimate of emitter location. The location estimate can be obtained from the least squares solution of

$$
\mathbf{A}^{\prime} \mathbf{x}^{\prime}=\mathbf{b}^{\prime}
$$

where $\mathbf{A}^{\prime}$ and $\mathbf{b}^{\prime}$ are defined as

$$
\begin{gathered}
\mathbf{A}^{\prime}=\left[\begin{array}{cc}
\sin \left(\phi_{1}+\delta \phi_{1}\right) & -\cos \left(\phi_{1}+\delta \phi_{1}\right) \\
\sin \left(\phi_{2}+\delta \phi_{2}\right) & -\cos \left(\phi_{2}+\delta \phi_{2}\right) \\
\vdots & \vdots \\
\sin \left(\phi_{N}+\delta \phi_{N}\right) & -\cos \left(\phi_{N}+\delta \phi_{N}\right)
\end{array}\right], \\
\mathbf{b}^{\prime}=\left[\begin{array}{c}
x_{1} \sin \left(\phi_{1}+\delta \phi_{1}\right)-y_{1} \cos \left(\phi_{1}+\delta \phi_{1}\right) \\
x_{2} \sin \left(\phi_{2}+\delta \phi_{2}\right)-y_{2} \cos \left(\phi_{2}+\delta \phi_{2}\right) \\
\vdots \\
x_{N} \sin \left(\phi_{N}+\delta \phi_{N}\right)-y_{N} \cos \left(\phi_{N}+\delta \phi_{N}\right)
\end{array}\right] .
\end{gathered}
$$

The normal equation of (7) is given by

$$
\mathbf{A}^{\prime H} \mathbf{A}^{\prime} \mathbf{x}^{\prime}=\mathbf{A}^{\prime H} \mathbf{b}^{\prime} \text {. }
$$

The location estimate is given by

$$
\mathbf{x}^{\prime}=\left(\mathbf{A}^{\prime H} \mathbf{A}^{\prime}\right)^{-1} \mathbf{A}^{\prime H} \mathbf{b}^{\prime}
$$

\section{Approximation of $\mathbf{A}^{\prime H} \mathbf{A}^{\prime}$ and $\mathbf{A}^{\prime H} \mathbf{b}^{\prime}$}

From (6) and (7), it is easy to show that the entries of $\mathbf{A}^{\prime H} \mathbf{A}^{\prime}$ and $\mathbf{A}^{\prime H} \mathbf{b}^{\prime}$ can be expressed as

$$
\left(\mathbf{A}^{\prime H} \mathbf{A}^{\prime}\right)_{11}=\sum_{i=1}^{N} \sin ^{2}\left(\phi_{i}+\delta \phi_{i}\right)
$$

$\left(\mathbf{A}^{\prime H} \mathbf{A}^{\prime}\right)_{12}=\left(\mathbf{A}^{\prime H} \mathbf{A}^{\prime}\right)_{21}=-\frac{1}{2} \sum_{i=1}^{N} \sin 2\left(\phi_{i}+\delta \phi_{i}\right)$, 


$$
\begin{aligned}
\left(\mathbf{A}^{\prime H} \mathbf{A}^{\prime}\right)_{22} & =\sum_{i=1}^{N} \cos ^{2}\left(\phi_{i}+\delta \phi_{i}\right), \\
\left(\mathbf{A}^{\prime H} \mathbf{b}^{\prime}\right)_{1} & =\sum_{i=1}^{N}\left(x_{i} \sin ^{2}\left(\phi_{i}+\delta \phi_{i}\right)-\frac{1}{2} y_{i} \sin 2\left(\phi_{i}+\delta \phi_{i}\right)\right), \\
\left(\mathbf{A}^{\prime H} \mathbf{b}^{\prime}\right)_{2} & =-\sum_{i=1}^{N}\left(\frac{1}{2} x_{i} \sin 2\left(\phi_{i}+\delta \phi_{i}\right)-y_{i} \cos ^{2}\left(\phi_{i}+\delta \phi_{i}\right)\right),
\end{aligned}
$$

where $\left(\mathbf{A}^{\prime H} \mathbf{A}^{\prime}\right)_{i j}$ and $\left(\mathbf{A}^{\prime H} \mathbf{b}^{\prime}\right)_{i j}$ denote the entry at the $i$ th row and the $j$ th column of $\mathbf{A}^{\prime H} \mathbf{A}^{\prime}$ and $\mathbf{A}^{\prime H} \mathbf{b}^{\prime}$, respectively. Note that $\mathbf{A}^{\prime H} \mathbf{A}^{\prime}$ is a $2 \times 2$ matrix and that $\mathbf{A}^{\prime H} \mathbf{b}^{\prime}$ is a $2 \times 1$ matrix. Let $\left(\mathbf{A}^{\prime H} \mathbf{A}^{\prime}\right)^{(k)}$ and $\left(\mathbf{A}^{\prime H} \mathbf{b}^{\prime}\right)^{(k)}$ denote the approximations of $\mathbf{A}^{\prime H} \mathbf{A}^{\prime}$ and $\mathbf{A}^{\prime H} \mathbf{b}^{\prime}$ based on the $k$ th-order Taylor series expansion, respectively:

$$
\begin{aligned}
& \mathbf{A}^{\prime H} \mathbf{A}^{\prime} \approx\left(\mathbf{A}^{\prime H} \mathbf{A}^{\prime}\right)^{(k)}=\mathbf{A}^{H} \mathbf{A}+\delta\left(\mathbf{A}^{H} \mathbf{A}\right)^{(k)}, \\
& \mathbf{A}^{\prime H} \mathbf{b}^{\prime} \approx\left(\mathbf{A}^{\prime H} \mathbf{b}^{\prime}\right)^{(k)}=\mathbf{A}^{H} \mathbf{b}+\delta\left(\mathbf{A}^{H} \mathbf{b}\right)^{(k)},
\end{aligned}
$$

where $\delta\left(\mathbf{A}^{H} \mathbf{A}\right)^{(k)}$ and $\delta\left(\mathbf{A}^{H} \mathbf{b}\right)^{(k)}$ are defined from

$$
\begin{aligned}
& \delta\left(\mathbf{A}^{H} \mathbf{A}\right)^{(k)}=\left(\mathbf{A}^{H} \mathbf{A}\right)^{(k)}-\mathbf{A}^{H} \mathbf{A}, \\
& \delta\left(\mathbf{A}^{H} \mathbf{b}\right)^{(k)}=\left(\mathbf{A}^{H} \mathbf{b}\right)^{(k)}-\mathbf{A}^{H} \mathbf{b} .
\end{aligned}
$$

If the first-order $K$-approximation, corresponding to $k=1$, is applied, (11) and (12) can be written as

$$
\begin{aligned}
\mathbf{A}^{\prime H} \mathbf{A}^{\prime} & \approx \mathbf{A}^{H} \mathbf{A}+\delta\left(\mathbf{A}^{H} \mathbf{A}\right)^{(k=1)}, \\
\mathbf{A}^{\prime H} \mathbf{b}^{\prime} & \approx \mathbf{A}^{H} \mathbf{b}+\delta\left(\mathbf{A}^{H} \mathbf{b}\right)^{(k=1)} .
\end{aligned}
$$

The explicit expressions of $\delta\left(\mathbf{A}^{\prime H} \mathbf{A}^{\prime}\right)^{(k=1)}$ and $\delta$ $\left(\mathbf{A}^{\prime H} \mathbf{b}^{\prime}\right)^{(k=1)}$ based on the first-order Taylor series are derived in [16]

$$
\begin{aligned}
& \delta\left(\mathbf{A}^{H} \mathbf{A}\right)_{11}^{(k=1)}=\sum_{i=1}^{N} \delta \phi_{i} \sin 2 \phi_{i}, \\
& \delta\left(\mathbf{A}^{H} \mathbf{A}\right)_{12}^{(k=1)}=-\sum_{i=1}^{N} \delta \phi_{i} \cos 2 \phi_{i}, \\
& \delta\left(\mathbf{A}^{H} \mathbf{A}\right)_{22}^{(k=1)}=-\sum_{i=1}^{N} \delta \phi_{i} \sin 2 \phi_{i},
\end{aligned}
$$

$$
\begin{aligned}
& \delta\left(\mathbf{A}^{H} \mathbf{b}\right)_{1}^{(k=1)}=\sum_{i=1}^{N}\left(x_{i} \delta \phi_{i} \sin 2 \phi_{i}-y_{i} \delta \phi_{i} \cos 2 \phi_{i}\right), \\
& \delta\left(\mathbf{A}^{H} \mathbf{b}\right)_{2}^{(k=1)}=\sum_{i=1}^{N}-\left(x_{i} \delta \phi_{i} \cos 2 \phi_{i}+y_{i} \delta \phi_{i} \sin 2 \phi_{i}\right) .
\end{aligned}
$$

The explicit expressions of $\delta\left(\mathbf{A}^{\prime H} \mathbf{A}^{\prime}\right)^{(k=2)}, \delta\left(\mathbf{A}^{\prime H} \mathbf{A}^{\prime}\right)^{(k=3)}$, $\delta\left(\mathbf{A}^{\prime H} \mathbf{b}^{\prime}\right)^{(k=2)}$, and $\delta\left(\mathbf{A}^{\prime H} \mathbf{b}^{\prime}\right)^{(k=3)}$ are derived in Appendices $\mathrm{A}$ and $\mathrm{B}$, where $k=2,3$ denotes that the second- and thirdorder Taylor expansion has been adopted.

\section{Error Bound for the $K$-Approximation}

In this section, we describe the error bound due to various orders of $K$-approximation for each of the entries of $\left(\mathbf{A}^{\prime H} \mathbf{A}^{\prime}\right)^{(k)}$ and $\left(\mathbf{A}^{\prime H} \mathbf{b}^{\prime}\right)^{(k)}$. In Table 1 , all the terms of Taylor series expansion for each entries of $\left(\mathbf{A}^{\prime H} \mathbf{A}^{\prime}\right)^{(k)}$ and $\left(\mathbf{A}^{\prime{ }^{H}} \mathbf{b}^{\prime}\right)^{(k)}$ are tabulated. Table 2 tabulates the first-order Taylor series, the second-order Taylor series, the third-order Taylor series, and the associated error bounds for each entry of $\left(\mathbf{A}^{\prime H} \mathbf{A}^{\prime}\right)_{11}^{(k)}$.

Note that the upper bound of absolute value of the error between the original function value and the $n$ th-order Taylor series is given by the $n+1$ th-order term of the Taylor series expansion. For example, the difference between the original function value and the first-order Taylor series is given by the second-order term of the Taylor series expansion, which is described in the second row of Table 2. The corresponding error bounds for the second-order Taylor series and the third-order Taylor series are given in the third row and the fourth row of Table 2, respectively.

Similarly, Tables 3-6 tabulate the same quantities for $\left(\mathbf{A}^{\prime H} \mathbf{A}^{\prime}\right)_{12}^{(k)}=\left(\mathbf{A}^{\prime H} \mathbf{A}^{\prime}\right)_{21}^{(k)}, \quad\left(\mathbf{A}^{\prime H} \mathbf{A}^{\prime}\right)_{22}^{(k)}, \quad\left(\mathbf{A}^{\prime H} \mathbf{b}^{\prime}\right)_{1}^{(k)}, \quad$ and $\left(\mathbf{A}^{\prime H} \mathbf{b}^{\prime}\right)_{2}^{(k)}$, respectively.

\section{Approximation of $\mathbf{x}^{\prime(k)}$}

Substituting (11) and (12) in (7) results in

$\left(\mathbf{A}^{H} \mathbf{A}+\delta\left(\mathbf{A}^{H} \mathbf{A}\right)^{(k)}\right) \mathbf{x}^{\prime(k)}=\mathbf{A}^{H} \mathbf{b}+\delta\left(\mathbf{A}^{H} \mathbf{b}\right)^{(k)}$,

$\mathbf{x}^{\prime(k)}=\left(\mathbf{A}^{H} \mathbf{A}+\delta\left(\mathbf{A}^{H} \mathbf{A}\right)^{(k)}\right)^{-1}\left(\mathbf{A}^{H} \mathbf{b}+\delta\left(\mathbf{A}^{H} \mathbf{b}\right)^{(k)}\right)$.

$L$-approximations of $\mathbf{x}^{\prime(k=1)}$ is denoted by $\mathbf{x}^{\prime(k=1, l=1)}$. In Appendix C, based on the perturbation of the solution of linear system, it is shown that $\mathbf{x}^{\prime(k, l=1)}$ can be written as

$\mathbf{x}^{\prime(k, l=1)}=\mathbf{x}_{T}+\left(\mathbf{A}^{H} \mathbf{A}\right)^{-1}\left(\delta\left(\mathbf{A}^{H} \mathbf{b}\right)^{(k)}-\delta\left(\mathbf{A}^{H} \mathbf{A}\right)^{(k)} \mathbf{x}_{T}\right)$. 
TABLE 1: Explicit expressions of each term of the Taylor series expansion.

\begin{tabular}{|c|c|c|c|c|c|c|}
\hline & Constant & First-order term & & Second-order term & Third-order term & Fourth-order term \\
\hline$\left(\mathbf{A}^{\prime H} \mathbf{A}^{\prime}\right)_{11}^{(k)}$ & $\sum_{i=1}^{N} \sin ^{2} \phi_{i}$ & $\sum_{i=1}^{N} \sin 2 \phi_{i} \delta \phi_{i}$ & & $\sum_{i=1}^{N} \cos 2 \phi_{i} \delta \phi_{i}^{2}$ & $-\frac{2}{3} \sum_{i=1}^{N} \sin 2 \phi_{i} \delta \phi_{i}^{3}$ & $-\frac{1}{3} \sum_{i=1}^{N} \cos 2 \phi_{i} \delta \phi_{i}$ \\
\hline$\left(\mathbf{A}^{\prime H} \mathbf{A}^{\prime}\right)_{12}^{(k)}$ & $-\frac{1}{2} \sum_{i=1}^{N} \sin 2 \phi_{i}$ & $-\sum_{i=1}^{N} \cos 2 \phi_{i} \delta \phi_{i}$ & & $\sum_{i=1}^{N} \sin 2 \phi_{i} \delta \phi_{i}^{2}$ & $\frac{2}{3} \sum_{i=1}^{N} \cos 2 \phi_{i} \delta \phi_{i}^{3}$ & $\frac{1}{3} \sum_{i=1}^{N} \sin 2 \phi_{i} \delta \phi_{i}^{4}$ \\
\hline$\left(\mathbf{A}^{\prime H} \mathbf{A}^{\prime}\right)_{22}^{(k)}$ & $\sum_{i=1}^{N} \cos ^{2} \phi_{i}$ & $-\sum_{i=1}^{N} \sin 2 \phi_{i} \delta \phi_{i}$ & & $-\sum_{i=1}^{N} \cos 2 \phi_{i} \delta \phi_{i}^{2}$ & $\frac{2}{3} \sum_{i=1}^{N} \sin 2 \phi_{i} \delta \phi_{i}^{3}$ & $\frac{1}{3} \sum_{i=1}^{N} \cos 2 \phi_{i} \delta \phi_{i}^{4}$ \\
\hline$\left(\mathbf{A}^{\prime H} \mathbf{b}^{\prime}\right)_{1}^{(k)}$ & $\sum_{i=1}^{N}\left(\begin{array}{c}x_{i} \sin ^{2} \phi_{i} \\
-\frac{y_{i}}{2} \sin 2 \phi_{i}\end{array}\right)$ & $\sum_{i=1}^{N}\left(\begin{array}{c}x_{i} \sin 2 \phi_{i} \\
-y_{i} \cos 2 \phi_{i}\end{array}\right) \delta$ & $\delta \phi_{i}$ & $\sum_{i=1}^{N}\left(\begin{array}{c}x_{i} \cos 2 \phi_{i} \\
+y_{i} \sin 2 \phi_{i}\end{array}\right) \delta \phi_{i}^{2}$ & $-\frac{2}{3} \sum_{i=1}^{N}\left(\begin{array}{c}-x_{i} \sin 2 \phi_{i} \\
+y_{i} \cos 2 \phi_{i}\end{array}\right) \delta \phi_{i}^{3}$ & $-\frac{1}{3} \sum_{i=1}^{N}\left(\begin{array}{c}x_{i} \cos 2 \phi_{i} \\
+y_{i} \sin 2 \phi_{i}\end{array}\right)$ \\
\hline$\left(\mathbf{A}^{\prime H} \mathbf{b}^{\prime}\right)^{(k)}$ & $-\sum_{i=1}^{N}\left(\begin{array}{c}\frac{x_{i}}{2} \sin 2 \phi_{i} \\
+y_{i} \cos ^{2} \phi_{i}\end{array}\right)$ & $-\sum_{i=1}^{N}\left(\begin{array}{c}x_{i} \cos 2 \phi_{i} \\
-y_{i} \sin 2 \phi_{i}\end{array}\right)$ & $\delta \phi_{i}$ & $\sum_{i=1}^{N}\left(\begin{array}{c}x_{i} \sin 2 \phi_{i} \\
+y_{i} \cos 2 \phi_{i}\end{array}\right) \delta \phi_{i}^{2}$ & $\frac{2}{3} \sum_{i=1}^{N}\left(\begin{array}{c}x_{i} \cos 2 \phi_{i} \\
+y_{i} \sin 2 \phi_{i}\end{array}\right) \delta \phi_{i}^{3}$ & $-\frac{1}{3} \sum_{i=1}^{N}\left(\begin{array}{c}x_{i} \sin 2 \phi_{i} \\
+y_{i} \cos 2 \phi_{i}\end{array}\right)$ \\
\hline
\end{tabular}

TABLe 2: Taylor series and error bound for $\left(\mathbf{A}^{\prime H} \mathbf{A}^{\prime}\right)_{11}^{(k)}$.

\begin{tabular}{|c|c|c|c|c|}
\hline & $\begin{array}{ccc}\text { Constant } & \text { First-order term } & \text { Second-order term } \\
\sum_{i=1}^{N} \sin ^{2} \phi_{i} & \sum_{i=1}^{N} \sin 2 \phi_{i} \delta \phi_{i} & \sum_{i=1}^{N} \cos 2 \phi_{i} \delta \phi_{i}{ }^{2}\end{array}$ & $\begin{array}{l}\text { Third-order term } \\
-\frac{2}{3} \sum_{i=1}^{N} \sin 2 \phi_{i} \delta \phi_{i}^{3}\end{array}$ & $\begin{array}{l}\text { Fourth-order term } \\
-\frac{1}{3} \sum_{i=1}^{N} \cos 2 \phi_{i} \delta \phi_{i}{ }^{4}\end{array}$ & Error bound \\
\hline First-order Taylor series & $\sum_{i=1}^{N} \sin ^{2} \phi_{i}+\sum_{i=1}^{N} \sin 2 \phi_{i} \delta \phi_{i}$ & & & $\left|\sum_{i=1}^{N} \cos 2 \phi_{i} \delta \phi_{i}^{2}\right|$ \\
\hline Second-order Taylor series & $\sum_{i=1}^{N} \sin ^{2} \phi_{i}+\sum_{i=1}^{N} \sin 2 \phi_{i} \delta \phi_{i}+\sum_{i=1}^{N} \cos 2 \phi_{i} \delta \phi_{i}^{2}$ & & & $\left|-\frac{2}{3} \sum_{i=1}^{N} \sin 2 \phi_{i} \delta \phi_{i}^{3}\right|$ \\
\hline Third-order Taylor series & $\sum_{i=1}^{N} \sin ^{2} \phi_{i}+\sum_{i=1}^{N} \sin 2 \phi_{i} \delta \phi_{i}+\sum_{i=1}^{N} \cos 2 \phi_{i} \delta \phi_{i}^{2}-$ & $\frac{2}{3} \sum_{i=1}^{N} \sin 2 \phi_{i} \delta \phi_{i}^{3}$ & & $\left|-\frac{1}{3} \sum_{i=1}^{N} \cos 2 \phi_{i} \delta \phi_{i}^{4}\right|$ \\
\hline
\end{tabular}

TABle 3: Taylor series and error bound for $\left(\mathbf{A}^{\prime H} \mathbf{A}^{\prime}\right)_{12}^{(k)}$.

\begin{tabular}{|c|c|c|c|c|}
\hline & $\begin{array}{ccc}\text { Constant } & \text { First-order-term } & \text { Second-order-term } \\
-\frac{1}{2} \sum_{i=1}^{N} \sin 2 \phi_{i} & -\sum_{i=1}^{N} \cos 2 \phi_{i} \delta \phi_{i} & \sum_{i=1}^{N} \sin 2 \phi_{i} \delta \phi_{i}^{2}\end{array}$ & $\begin{array}{l}\text { Third-order term } \\
\frac{2}{3} \sum_{i=1}^{N} \cos 2 \phi_{i} \delta \phi_{i}^{3}\end{array}$ & $\begin{array}{l}\text { Fourth-order term } \\
\frac{1}{3} \sum_{i=1}^{N} \sin 2 \phi_{i} \delta \phi_{i}^{4}\end{array}$ & Error bound \\
\hline First-order Taylor series & $-\frac{1}{2} \sum_{i=1}^{N} \sin 2 \phi_{i}-\sum_{i=1}^{N} \cos 2 \phi_{i} \delta \phi_{i}$ & & & $\left|\sum_{i=1}^{N} \sin 2 \phi_{i} \delta \phi_{i}^{2}\right|$ \\
\hline $\begin{array}{l}\text { Second-order Taylor } \\
\text { series }\end{array}$ & $-\frac{1}{2} \sum_{i=1}^{N} \sin 2 \phi_{i}-\sum_{i=1}^{N} \cos 2 \phi_{i} \delta \phi_{i}+\sum_{i=1}^{N} \sin 2 \phi_{i} \delta \phi_{i}^{2}$ & & & $\frac{2}{3} \sum_{i=1}^{N} \cos 2 \phi_{i} \delta \phi_{i}^{3}$ \\
\hline Third-order Taylor series & $-\frac{1}{2} \sum_{i=1}^{N} \sin 2 \phi_{i}-\sum_{i=1}^{N} \cos 2 \phi_{i} \delta \phi_{i}+\sum_{i=1}^{N} \sin 2 \phi_{i} \delta \phi_{i}^{2}+$ & $\frac{2}{3} \sum_{i=1}^{N} \cos 2 \phi_{i} \delta \phi_{i}^{3}$ & & $\left|\frac{1}{3} \sum_{i=1}^{N} \sin 2 \phi_{i} \delta \phi_{i}{ }^{4}\right|$ \\
\hline
\end{tabular}

\section{Analytic Expression of the MSE of the Location Estimate}

In this section, we derive explicit closed-form expressions of the mean-squared error (MSE) of the location estimates in (18). Let $x_{T}$ and $y_{T}$ denote the $x$-coordinate and $y$-coordi- nate of $\mathbf{x}_{T}$, respectively:

$$
\mathbf{x}_{T}=\left[\begin{array}{ll}
x_{T} & y_{T}
\end{array}\right]^{T}
$$

Similarly, $x^{\prime(k, l=1)}$ and $y^{\prime(k, l=1)}$ denote the $x$-coordinate 
TABLE 4: Taylor series and error bound for $\left(\mathbf{A}^{\prime H} \mathbf{A}^{\prime}\right)_{22}^{(k)}$.

Constant First-order term Second-order term Third-order term Fourth-order term $\sum_{i=1}^{N} \cos ^{2} \phi_{i}-\sum_{i=1}^{N} \sin 2 \phi_{i} \delta \phi_{i} \quad-\sum_{i=1}^{N} \cos 2 \phi_{i} \delta \phi_{i}{ }^{2} \quad \frac{2}{3} \sum_{i=1}^{N} \sin 2 \phi_{i} \delta \phi_{i}{ }^{3} \quad \frac{1}{3} \sum_{i=1}^{N} \cos 2 \phi_{i} \delta \phi_{i}{ }^{4} \quad$ Error bound

First-order Taylor series $\quad \sum_{i=1}^{N} \cos ^{2} \phi_{i}-\sum_{i=1}^{N} \sin 2 \phi_{i} \delta \phi_{i} \quad\left|-\sum_{i=1}^{N} \cos 2 \phi_{i} \delta \phi_{i}^{2}\right|$

Second-order Taylor series $\quad \sum_{i=1}^{N} \cos ^{2} \phi_{i}-\sum_{i=1}^{N} \sin 2 \phi_{i} \delta \phi_{i}-\sum_{i=1}^{N} \cos 2 \phi_{i} \delta \phi_{i}^{2} \quad\left|\frac{2}{3} \sum_{i=1}^{N} \sin 2 \phi_{i} \delta \phi_{i}{ }^{3}\right|$

Third-order Taylor series $\quad \sum_{i=1}^{N} \cos ^{2} \phi_{i}-\sum_{i=1}^{N} \sin 2 \phi_{i} \delta \phi_{i}-\sum_{i=1}^{N} \cos 2 \phi_{i} \delta \phi_{i}{ }^{2}+\frac{2}{3} \sum_{i=1}^{N} \sin 2 \phi_{i} \delta \phi_{i}{ }^{3} \quad\left|\frac{1}{3} \sum_{i=1}^{N} \cos 2 \phi_{i} \delta \phi_{i}{ }^{4}\right|$

and $y$-coordinate of $\mathbf{x}^{\prime(k, l=1)}$, respectively:

$$
\mathbf{x}^{\prime(k=1, l=1)}=\left[\begin{array}{ll}
x^{\prime(k, l=1)} & y^{\prime(k, l=1)}
\end{array}\right]^{T} .
$$

Euclidean distance between $\mathbf{x}^{\prime}$ and $\mathbf{x}_{T}$ is given by

$$
d\left(\mathbf{x}^{\prime}, \mathbf{x}_{T}\right)=\left\|\mathbf{x}^{\prime}-\mathbf{x}_{T}\right\|_{2}
$$

Similarly, the distance between $\mathbf{x}^{\prime(k, l=1)}$ and $\mathbf{x}_{T}$ is written as

$$
d\left(\mathbf{x}^{\prime(k, l=1)}, \mathbf{x}_{T}\right)=\left\|\mathbf{x}^{\prime(k, l=1)}-\mathbf{x}_{T}\right\|_{2} .
$$

Substituting (18) in (22) yields

$$
\left\|\mathbf{x}^{\prime(k, l=1)}-\mathbf{x}_{T}\right\|_{2}=\left\|\left(\mathbf{A}^{H} \mathbf{A}\right)^{-1}\left(\delta\left(\mathbf{A}^{H} \mathbf{b}\right)^{(k)}-\delta\left(\mathbf{A}^{H} \mathbf{A}\right)^{(k)} \mathbf{x}_{T}\right)\right\|_{2} .
$$

From (23), the MSE of the location estimate is

$$
\begin{aligned}
E\left(\left\|\mathbf{x}^{\prime(k, l=1)}-\mathbf{x}_{T}\right\|_{2}^{2}\right) & \\
= & E\left(\left(\delta\left(\mathbf{A}^{H} \mathbf{b}\right)^{(k)}-\delta\left(\mathbf{A}^{H} \mathbf{A}\right)^{(k)} \mathbf{x}_{T}\right)^{H}\left(\left(\mathbf{A}^{H} \mathbf{A}\right)^{-1}\right)^{H}\right. \\
& \left.\cdot\left(\mathbf{A}^{H} \mathbf{A}\right)^{-1}\left(\delta\left(\mathbf{A}^{H} \mathbf{b}\right)^{(k)}-\delta\left(\mathbf{A}^{H} \mathbf{A}\right)^{(k)} \mathbf{x}_{T}\right)\right) .
\end{aligned}
$$

Let $\mathbf{D}$ and $\mathbf{H}$ be defined as

$$
\begin{aligned}
\mathbf{D} & \equiv\left(\delta\left(\mathbf{A}^{H} \mathbf{b}\right)^{(k)}-\delta\left(\mathbf{A}^{H} \mathbf{A}\right)^{(k)} \mathbf{x}_{T}\right) \\
& =\left[\begin{array}{c}
\delta\left(\mathbf{A}^{H} \mathbf{b}\right)_{1}^{(k)}-\left(\delta\left(\mathbf{A}^{H} \mathbf{A}\right)_{11}^{(k)} x_{T}+\delta\left(\mathbf{A}^{H} \mathbf{A}\right)_{12}^{(k)} y_{T}\right) \\
\delta\left(\mathbf{A}^{H} \mathbf{b}\right)_{2}^{(k)}-\left(\delta\left(\mathbf{A}^{H} \mathbf{A}\right)_{21}^{(k)} x_{T}+\delta\left(\mathbf{A}^{H} \mathbf{A}\right)_{22}^{(k)} y_{T}\right)
\end{array}\right],
\end{aligned}
$$

$$
\mathbf{H} \equiv\left(\left(\mathbf{A}^{H} \mathbf{A}\right)^{-1}\right)^{\mathrm{T}}\left(\mathbf{A}^{H} \mathbf{A}\right)^{-1}=\left[\begin{array}{ll}
\left(\mathbf{A}^{H} \mathbf{A}\right)_{11}^{-1} & \left(\mathbf{A}^{H} \mathbf{A}\right)_{12}^{-1} \\
\left(\mathbf{A}^{H} \mathbf{A}\right)_{21}^{-1} & \left(\mathbf{A}^{H} \mathbf{A}\right)_{22}^{-1}
\end{array}\right]^{2}
$$

In terms of $\mathbf{D}$ in (25) and $\mathbf{H}$ in (26), the MSE in (24) can be rewritten as

$$
\begin{aligned}
& E\left(\left\|\mathbf{x}^{\prime(k, l=1)}-\mathbf{x}_{T}\right\|_{2}^{2}\right) \\
&= E\left(\mathbf{D}^{T} \mathbf{H D}\right)=H_{11} E\left(D_{1}^{2}\right) \\
&+\left(H_{12}+H_{21}\right) E\left(D_{1} D_{2}\right)+H_{22} E\left(D_{2}^{2}\right) \\
&= \sum_{p=1}^{2} \sum_{q=1}^{2} H_{p q} E\left[D_{p} D_{q}\right] .
\end{aligned}
$$

where $D_{i}$ denote and the $i$ th entry of $\mathbf{D}$ and $H_{i j}$ denote the entry at the $i$ th row and thejth column of $\mathbf{H}$, respectively.

$E\left(D_{1}^{2}\right), E\left(D_{1} D_{2}\right)$, and $E\left(D_{2}^{2}\right)$ are explicitly expressed as

$$
\begin{aligned}
E\left(D_{1}^{2}\right)= & E\left(\left(\delta\left(\mathbf{A}^{H} \mathbf{b}\right)_{1}^{(k)}\right)^{2}\right)+x_{T} E\left(\left(\delta\left(\mathbf{A}^{H} \mathbf{A}\right)_{11}^{(k)}\right)^{2}\right) \\
& +y_{T}^{2} E\left(\left(\delta\left(\mathbf{A}^{H} \mathbf{A}\right)_{12}^{(k)}\right)^{2}\right) \\
& +2 x_{T} y_{T} E\left(\delta\left(\mathbf{A}^{H} \mathbf{A}\right)_{11}^{(k)} \delta\left(\mathbf{A}^{H} \mathbf{A}\right)_{12}^{(k)}\right. \\
& -2 x_{T} E\left(\delta\left(\mathbf{A}^{H} \mathbf{b}\right)_{1}^{(k)} \delta\left(\mathbf{A}^{H} \mathbf{A}\right)_{11}^{(k)}\right) \\
& -2 y_{T} E\left(\delta\left(\mathbf{A}^{H} \mathbf{b}\right)_{1}^{(k)} \delta\left(\mathbf{A}^{H} \mathbf{A}\right)_{12}^{(k)}\right)
\end{aligned}
$$




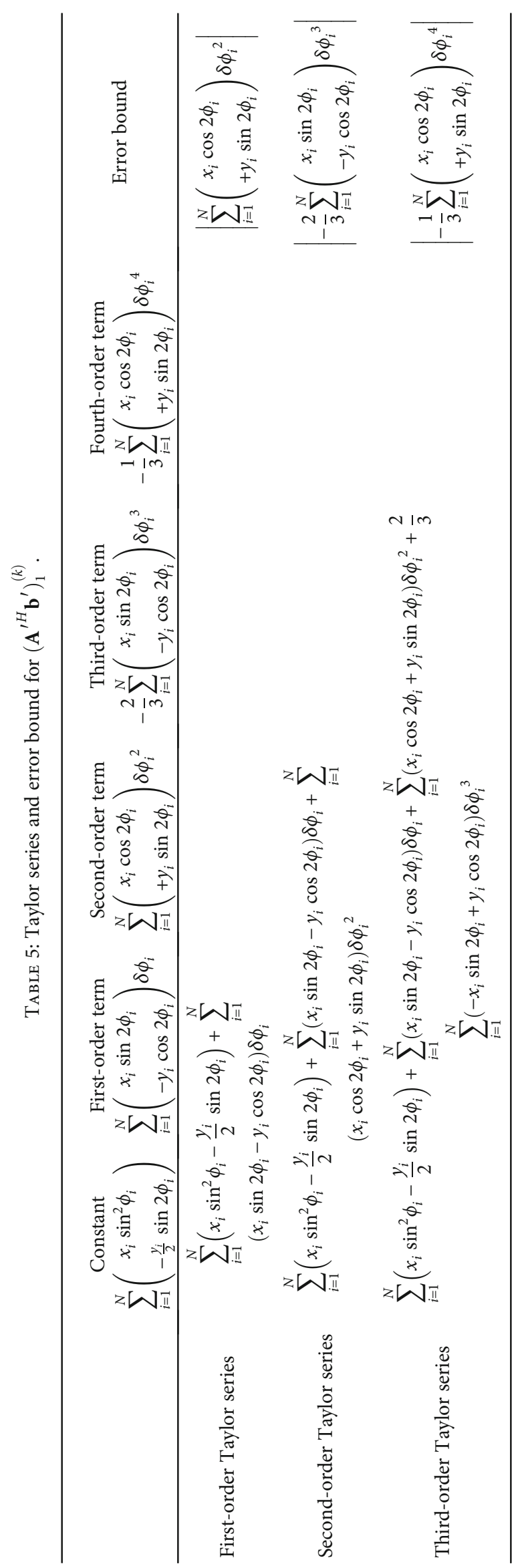




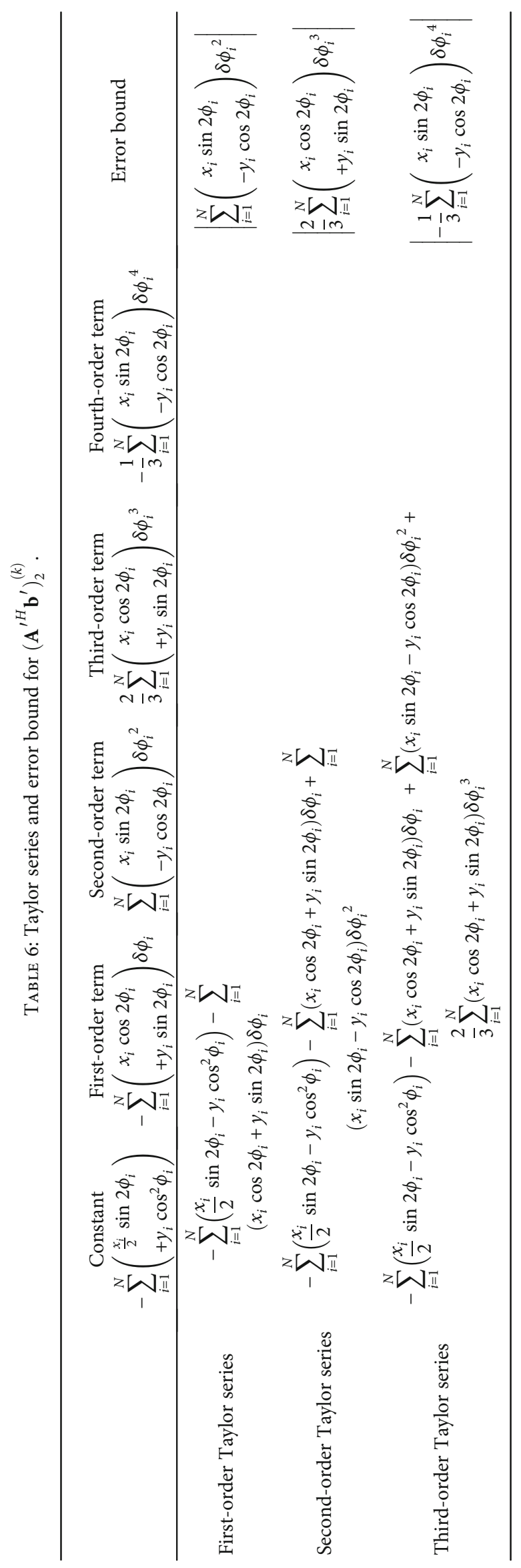


TABLE 7: Summary of the performance analysis of the AOA-based localization algorithm using the LS approach.

\begin{tabular}{lc}
\hline Data & Estimate of emitter location \\
\hline $\mathbf{A}^{H} \mathbf{A}, \mathbf{A}^{H} \mathbf{b}$ & $\mathbf{x}_{T}=\left(\mathbf{A}^{H} \mathbf{A}\right)^{-1} \mathbf{A}^{H} \mathbf{b}$ \\
$\mathbf{A}^{\prime H} \mathbf{A}^{\prime}, \mathbf{A}^{\prime H} \mathbf{b}^{\prime}$ & $\mathbf{x}^{\prime}=\left(\mathbf{A}^{\prime H} \mathbf{A}^{\prime}\right)^{-1} \mathbf{A}^{\prime H} \mathbf{b}^{\prime}$ \\
$\left(\mathbf{A}^{\prime H} \mathbf{A}^{\prime}\right)^{(k)}=\mathbf{A}^{H} \mathbf{A}+\delta\left(\mathbf{A}^{H} \mathbf{A}\right)^{(k)}$ & $\mathbf{x}^{\prime(k)}=\left(\left(\mathbf{A}^{\prime H} \mathbf{A}^{\prime}\right)^{(k)}\right)^{-1}\left(\mathbf{A}^{\prime H} \mathbf{b}^{\prime}\right)^{(k)}=\left(\left(\mathbf{A}^{H} \mathbf{A}\right)+\delta\left(\mathbf{A}^{H} \mathbf{A}\right)^{(k)}\right)^{-1}\left(\left(\mathbf{A}^{H} \mathbf{b}\right)^{(k)}+\delta\left(\mathbf{A}^{H} \mathbf{b}\right)^{(k)} \mathbf{x}_{T}\right)$ \\
$\left(\mathbf{A}^{\prime H} \mathbf{b}^{\prime}\right)^{(k)}=\mathbf{A}^{H} \mathbf{b}+\delta\left(\mathbf{A}^{H} \mathbf{b}\right)^{(k)}$ & $\mathbf{x}^{\prime(k, l=1)}=\mathbf{x}_{T}+\delta \mathbf{x}^{(k, l=1)}=\mathbf{x}_{\mathbf{T}}+\left(\mathbf{A}^{H} \mathbf{A}\right)^{-1}\left(\delta\left(\mathbf{A}^{H} \mathbf{b}\right)^{(k)}-\delta\left(\mathbf{A}^{H} \mathbf{A}\right)^{(k)} \mathbf{x}_{T}\right)$ \\
\hline
\end{tabular}

$$
\begin{aligned}
E\left(D_{1} D_{2}\right)= & E\left(\delta\left(\mathbf{A}^{H} \mathbf{b}\right)_{1}^{(k)} \delta\left(\mathbf{A}^{H} \mathbf{b}\right)_{2}^{(k)}\right) \\
& -x_{T} E\left(\delta\left(\mathbf{A}^{H} \mathbf{b}\right)_{1}^{(k)} \delta\left(\mathbf{A}^{H} \mathbf{A}\right)_{21}^{(k)}\right) \\
& -y_{T} E\left(\delta\left(\mathbf{A}^{H} \mathbf{b}\right)_{1}^{(k)} \delta\left(\mathbf{A}^{H} \mathbf{A}\right)_{22}^{(k)}\right) \\
& -x_{T} E\left(\delta\left(\mathbf{A}^{H} \mathbf{b}\right)_{2}^{(k)} \delta\left(\mathbf{A}^{H} \mathbf{A}\right)_{11}^{(k)}\right) \\
& -y_{T} E\left(\delta\left(\mathbf{A}^{H} \mathbf{b}\right)_{2}^{(k)} \delta\left(\mathbf{A}^{H} \mathbf{A}\right)_{12}^{(k)}\right) \\
& +x_{T}^{2} E\left(\delta\left(\mathbf{A}^{H} \mathbf{A}\right)_{11}^{(k)} \delta\left(\mathbf{A}^{H} \mathbf{A}\right)_{21}^{(k)}\right) \\
& +x_{T} y_{T} E\left(\delta\left(\mathbf{A}^{H} \mathbf{A}\right)_{11}^{(k)} \delta\left(\mathbf{A}^{H} \mathbf{A}\right)_{22}^{(k)}\right) \\
& +x_{T} y_{T} E\left(\delta\left(\mathbf{A}^{H} \mathbf{A}\right)_{12}^{(k)} \delta\left(\mathbf{A}^{H} \mathbf{A}\right)_{21}^{(k)}\right) \\
& +y_{T}^{2} E\left(\delta\left(\mathbf{A}^{H} \mathbf{A}\right)_{12}^{(k)} \delta\left(\mathbf{A}^{H} \mathbf{A}\right)_{22}^{(k)}\right) \\
E\left(D_{2}^{2}\right)= & E\left(\left(\delta\left(\mathbf{A}^{H} \mathbf{b}\right)_{2}^{(k)}\right)^{2}\right)+x_{T} E\left(\left(\delta\left(\mathbf{A}^{H} \mathbf{A}\right)_{21}^{(k)}\right)^{2}\right) \\
& +y_{T}^{2} E\left(\left(\delta\left(\mathbf{A}^{H} \mathbf{A}\right)_{22}^{(k)}\right)^{2}\right) \\
& +2 x_{T} y_{T} E\left(\delta\left(\mathbf{A}^{H} \mathbf{A}\right)_{21}^{(k)} \delta\left(\mathbf{A}^{H} \mathbf{A}\right)_{22}^{(k)}\right) \\
& -2 x_{T} E\left(\delta\left(\mathbf{A}^{H} \mathbf{b}\right)_{2}^{(k)} \delta\left(\mathbf{A}^{H} \mathbf{A}\right)_{21}^{(k)}\right) \\
& -2 y_{T} E\left(\delta\left(\mathbf{A}^{H} \mathbf{b}\right)_{2}^{(k)} \delta\left(\mathbf{A}^{H} \mathbf{A}\right)_{22}^{(k)}\right) \\
& (29)
\end{aligned}
$$

$\left(X_{1}, Y_{1}\right.$

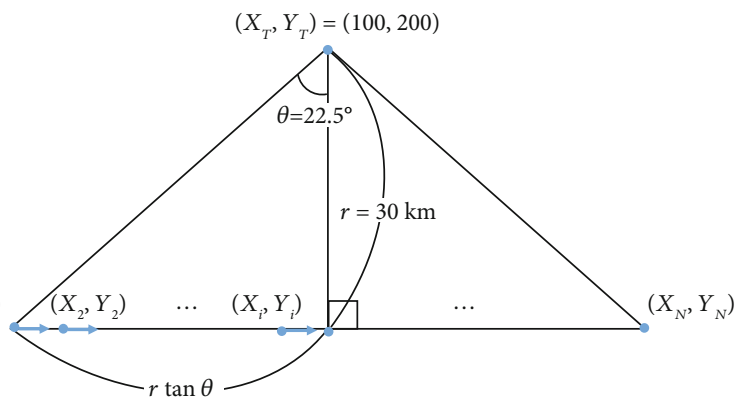

(a) Linear trajectory

$\left(X_{T}, Y_{T}\right)=(100,200)$

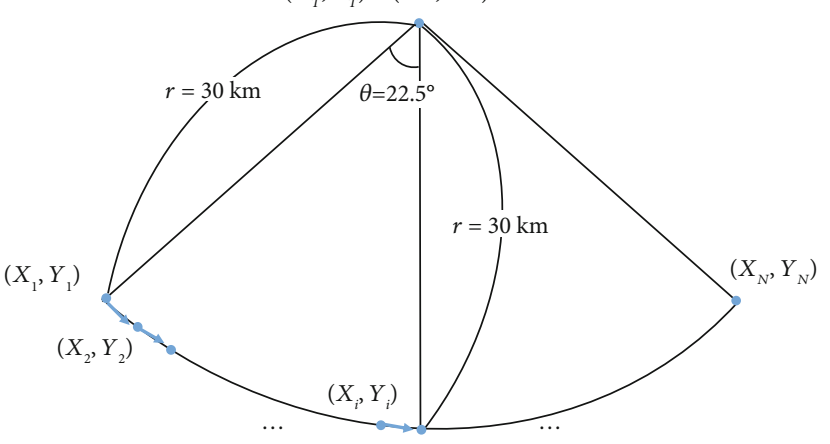

(b) Circular trajectory

Figure 1: Geometry of the localization.

The empirical MSE of $\mathbf{x}^{\prime}, \mathbf{x}^{\prime(k=1)}$ and $\mathbf{x}^{\prime(k=1, l=1)}$ is defined as

$$
\begin{aligned}
& \text { Simulation } E\left(\left\|\mathbf{x}^{\prime}-\mathbf{x}_{T}\right\|^{2}\right) \\
& \quad=\frac{1}{S} \sum_{s=1}^{S}\left(\left|x_{(s)}^{\prime}-x_{T}\right|^{2}+\left|y_{(s)}^{\prime}-y_{T}\right|^{2}\right),
\end{aligned}
$$

expansion, the second-order Taylor expansion, and thirdorder Taylor series have been employed in Appendices D, $\mathrm{E}$, and $\mathrm{F}$, respectively.

The summary of the localization algorithm is tabulated in Table 7.

\section{Results and Discussion}

Trajectory of sensor locations is given in Figure 1.

Simulation parameters are as follows:

Speed of sensor: $0.280 \mathrm{~km} / \mathrm{sec}$

Sampling interval: $2 \mathrm{sec}$

Number of sensor locations: 100

Number of Monte Carlo simulation: $S=1000$

$$
\begin{aligned}
& \text { Simulation } E\left(\left\|\mathbf{x}^{\prime(k=1)}-\mathbf{x}_{T}\right\|^{2}\right) \\
& =\frac{1}{S} \sum_{s=1}^{S}\left(\left|\mathbf{x}_{(s)}^{\prime(k=1)}-x_{T}\right|^{2}+\left|y_{(s)}^{\prime(k=1)}-y_{T}\right|^{2}\right),
\end{aligned}
$$

$$
\begin{aligned}
& \text { Simulation } E\left(\left\|\mathbf{x}^{\prime(k=1, l=1)}-\mathbf{x}_{T}\right\|^{2}\right) \\
& =\frac{1}{S} \sum_{s=1}^{S}\left(\left|x^{\prime(s)}{ }_{(s) 1, l=1)}^{(k)} x_{T}\right|^{2}+\left|y_{(s)}^{\prime(k=1, l=1)}-y_{T}\right|^{2}\right),
\end{aligned}
$$




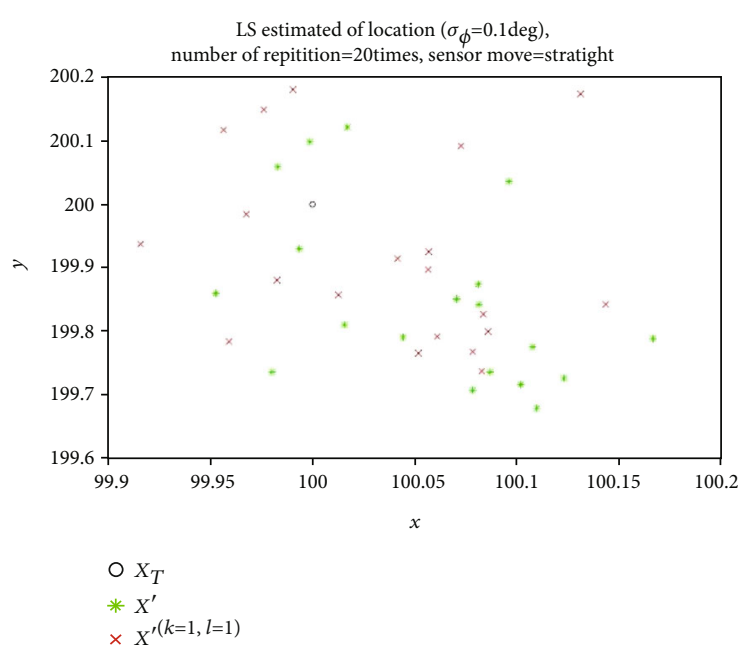

(a)

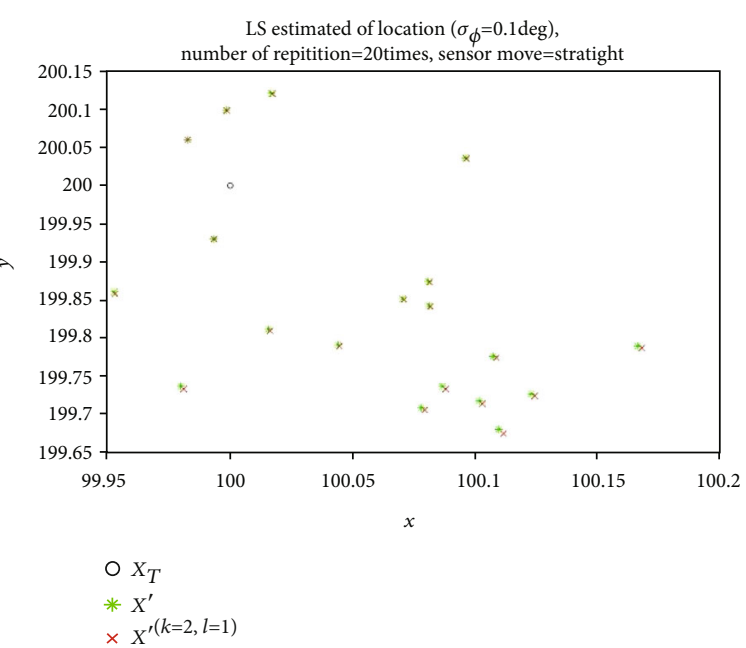

(b)

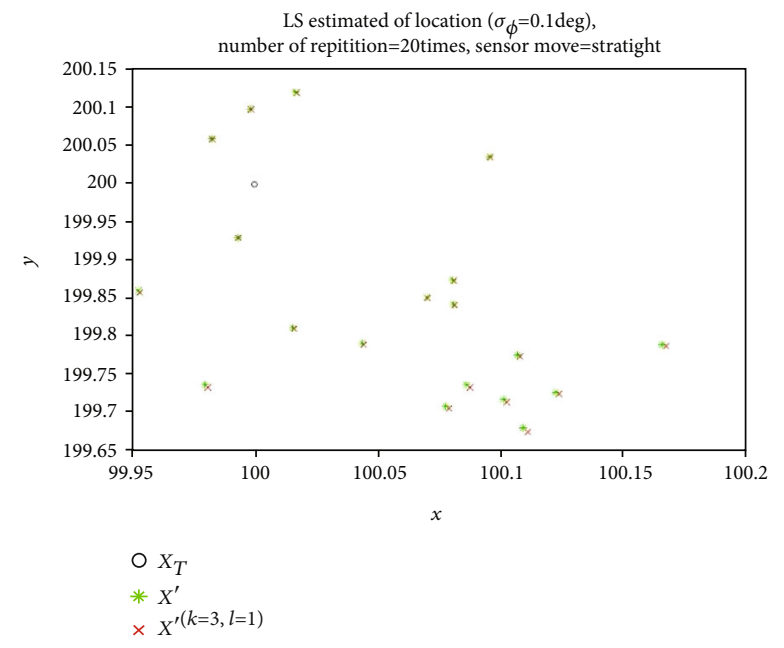

(c)

FIGURE 2: Distribution of estimates for the linear trajectory: (a) first-order $K$-approximation, (b) second-order $K$-approximation, and (c) third-order $K$-approximation.

where the lower script $(s)$ denotes the estimate associated with the $s$ th repetition out of $S$ repetitions.

Similarly, the empirical MSEs of $\mathbf{x}^{\prime(k=2)}, \mathbf{x}^{\prime(k=2, l=1)}, \mathbf{x}^{\prime(k=3)}$, and $\mathbf{x}^{\prime(k=3, l=1)}$ are given by

$$
\begin{aligned}
& \text { Simulation } E\left(\left\|\mathbf{x}^{\prime(k=2)}-\mathbf{x}_{T}\right\|^{2}\right) \\
& =\frac{1}{S} \sum_{s=1}^{S}\left(\left|x_{(s)}^{\prime(k=2)}-x_{T}\right|^{2}+\left|y^{\prime(s)}(k=2)-y_{T}\right|^{2}\right), \\
& \text { Simulation } E\left(\left\|\mathbf{x}^{\prime(k=2, l=1)}-\mathbf{x}_{T}\right\|^{2}\right) \\
& =\frac{1}{S} \sum_{s=1}^{S}\left(\left|x_{(s)}^{\prime(k=2, l=1)}-x_{T}\right|^{2}+\left|y_{(s)}^{\prime(k=2, l=1)}-y_{T}\right|^{2}\right),
\end{aligned}
$$

$$
\begin{aligned}
& \text { Simulation } E\left(\left\|\mathbf{x}^{(k=3)}-\mathbf{x}_{T}\right\|^{2}\right) \\
& =\frac{1}{S} \sum_{s=1}^{S}\left(\left|x^{\prime(s)}{ }_{(s=3)}^{(s)}-x_{T}\right|^{2}+\left|y_{(s)}^{\prime(k=3)}-y_{T}\right|^{2}\right), \\
& \text { Simulation } E\left(\left\|\mathbf{x}^{\prime(k=3, l=1)}-\mathbf{x}_{T}\right\|^{2}\right) \\
& =\frac{1}{S} \sum_{s=1}^{S}\left(\left|x_{(s)}^{\prime^{(k=3, l=1)}}-x_{T}\right|^{2}+\left|y_{(s)}^{\prime(k=3, l=1)}-y_{T}\right|^{2}\right) .
\end{aligned}
$$

$\mathbf{x}^{\prime}$ in (31) is defined in (9). $\mathbf{x}^{\prime(k=1)}$ and $\mathbf{x}^{\prime(k=1, l=1)}$ in (32) and (33) are given by (17) and (18) with $k=1$. Similarly, $\mathbf{x}^{\prime(k=2)}$ and $\mathbf{x}^{\prime(k=2, l=1)}$ in (34) and (35) are given by (17) and (18) with $k=2 . \mathbf{x}^{\prime^{(k=3)}}$ and $\mathbf{x}^{\prime^{(k=3, l=1)}}$ in (36) and (37) are given by (17) and (18) with $k=3$.

The results with $\mathbf{x}^{{ }^{(k=1, l=1)}}$ can be calculated using (9) and (18), respectively. In Figures 2(a)-2(c), we illustrate how the 


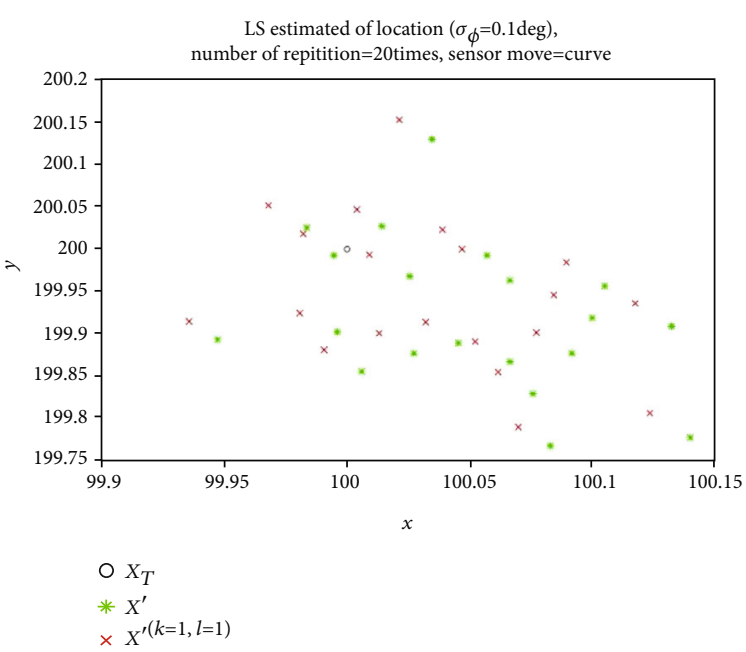

(a)

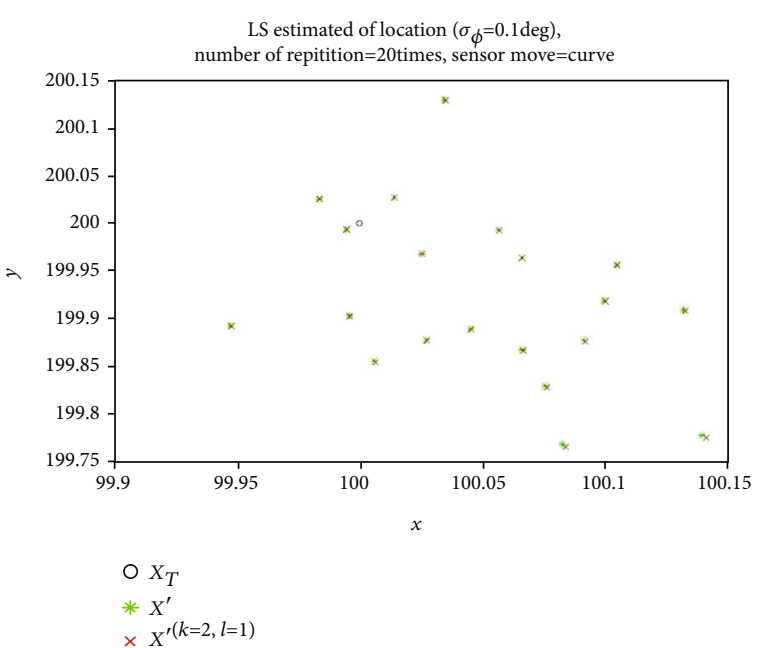

(b)

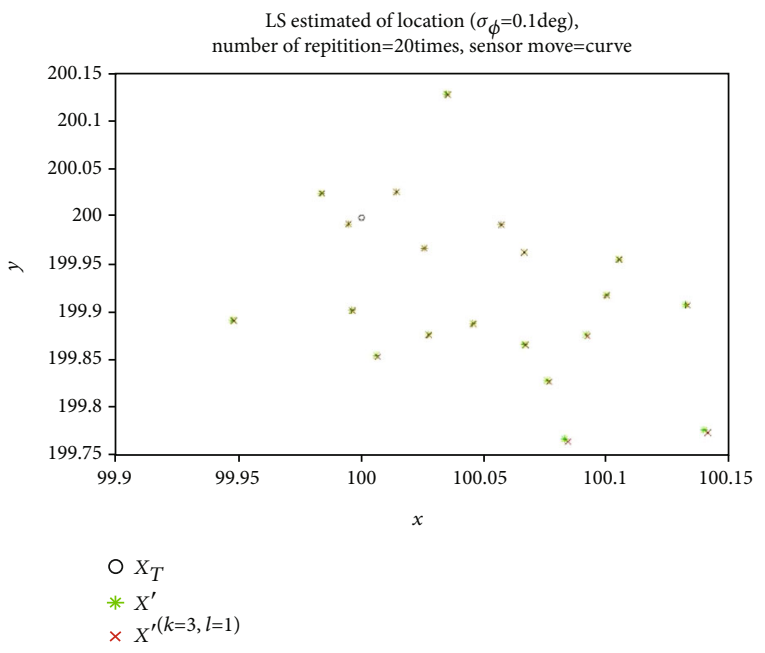

(c)

FIGURE 3: Distribution of estimates for the circular trajectory: (a) first-order $K$-approximation, (b) second-order $K$-approximation, and (c) third-order $K$-approximation.

location estimates are distributed with $k=1, k=2$, and $k=3$ for the linear trajectory. It is clear that $\mathbf{x}^{\prime(k=2, l=1)}$ is more accurate than $\mathbf{x}^{\prime(k=1, l=1)}$ and that $\mathbf{x}^{\prime(k=3, l=1)}$ is more accurate than $\mathbf{x}^{\prime(k=2, l=1)}$. Similarly, $\mathbf{x}^{\prime(k=2, l=1)}$ is closer to $\mathbf{x}^{\prime}$ than $\mathbf{x}^{\prime(k=1, l=1)}$ and $\mathbf{x}^{\prime(k=3, l=1)}$ is closer to $\mathbf{x}^{\prime}$ than $\mathbf{x}^{\prime(k=2, l=1)}$. Similar observations can be made in Figure 3, where the results for the circular trajectory have been illustrated.

Linear trajectory in Figure 1(a) is considered to get actual error and error bound in Figure 4. In Figure 4(a), the actual errors for $\left(\mathbf{A}^{\prime H} \mathbf{A}^{\prime}\right)_{11}^{(k)}$ and their error bounds associated with the first-order Taylor series for 50 repetitions are illustrated. Note that the $x$-axis represents each independent trial. It is clearly shown that the errors for all the cases are actually smaller than the error bounds. Figure 4(b) represents corresponding results for $\left(\mathbf{A}^{\prime H^{H}} \mathbf{b}^{\prime}\right)_{1}^{(k)}$, respectively.
The results validating the derived MSEs are illustrated in Figures 5 and 6 . The results in Figures 5 and 6 correspond to the linear trajectory in Figure 1(a) and the circular trajectory in Figure 1(b), respectively.

Standard deviations of AOA estimation error are set to 10 logarithmically equally spaced values between 0.01 and 10 degrees. More specifically, the standard deviations are approximately given by $0.01,0.0215,0.0464,0.1,0.2154$, $0.4642,1.0,2.1544,4.6416$, and 10 .

The MSEs with respect to the standard derivation of the LOB error are illustrated in Figures 5(a) and 5(b) for the linear trajectory. The results in Figure 5(a) with 'Simulation $E\left(\left\|\mathbf{x}^{\prime}-\mathbf{x}\right\|^{2}\right)^{\prime}$, 'Simulation $E\left(\left\|\mathbf{x}^{\prime(k=1)}-\mathbf{x}\right\|^{2}\right)^{\prime}$, 'Simulation $E\left(\left\|\mathbf{x}^{\prime(k=1, l=1)}-\mathbf{x}\right\|^{2}\right)^{\prime}$, and 'Analytic $E\left(\left\|\mathbf{x}^{\prime(k=1, l=1)}-\mathbf{x}\right\|^{2}\right)^{\prime}$ are obtained from (31), (32), (33) and (27), respectively. 


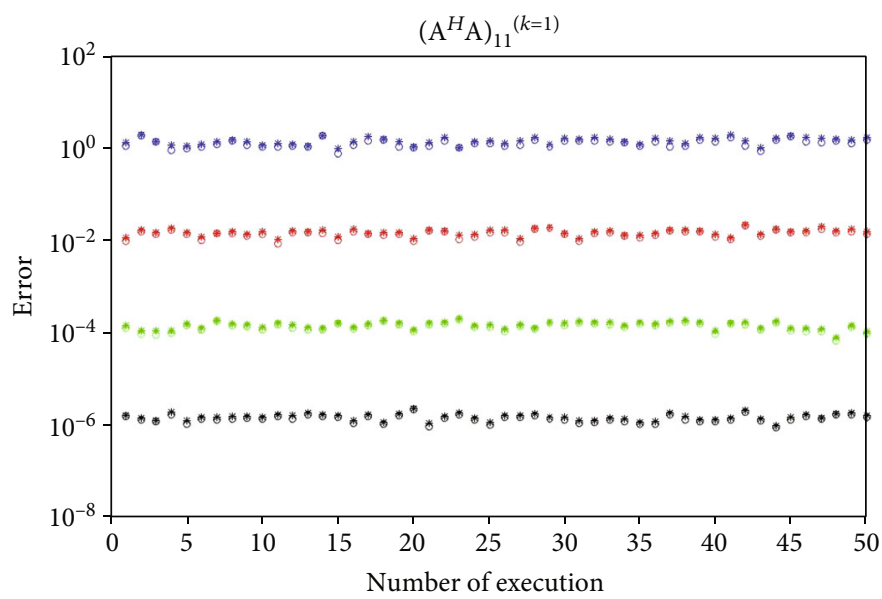

(a)

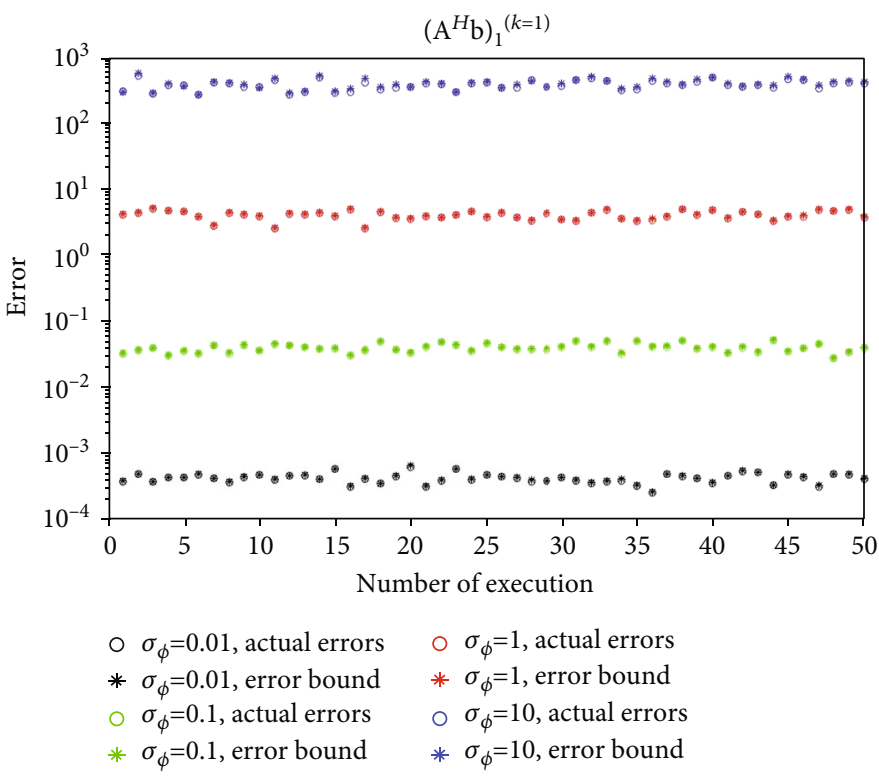

(b)

Figure 4: Error bound: (a) $\left(\mathbf{A}^{\prime H} \mathbf{A}^{\prime}\right)_{11}^{(k)}$; (b) $\left(\mathbf{A}^{\prime H} \mathbf{b}^{\prime}\right)_{1}^{(k)}$.

'Simulation $E\left(\left\|\mathbf{x}^{\prime}-\mathbf{x}\right\|^{2}\right)^{\prime}$ is not equal to 'Simulation $E\left(\left\|\mathbf{x}^{\prime(k=1)}-\mathbf{x}\right\|^{2}\right)^{\prime}$, since $K$-approximation is used to get 'Simulation $E\left(\left\|\mathbf{x}^{\prime(k=1)}-\mathbf{x}\right\|^{2}\right)^{\prime}$ from 'Simulation $E\left(\| \mathbf{x}^{\prime}\right.$ $\left.\mathbf{x} \|^{2}\right)^{\prime}$. Similarly, 'Simulation $E\left(\left\|\mathbf{x}^{\prime(k=1)}-\mathbf{x}\right\|^{2}\right)^{\prime}$ is not equal to 'Simulation $E\left(\left\|\mathbf{x}^{\prime(k=1, l=1)}-\mathbf{x}\right\|^{2}\right)^{\prime}$, since $L$-approximation is adopted to get 'Simulation $E\left(\left\|\mathbf{x}^{(k=1, l=1)}-\mathbf{x}\right\|^{2}\right)^{\prime}$ from ' Simulation $E\left(\left\|x^{\prime(k=1)}-x\right\|^{2}\right)^{\prime}$. Although, they are not exactly equal, it is clear from Figure 5 that 'Simulation $E\left(\left\|\mathbf{x}^{\prime}-\mathbf{x}\right\|^{2}\right)^{\prime}$ is approximately equal to 'Simulation $E$ $\left(\left\|\mathbf{x}^{\prime(k=1)}-\mathbf{x}\right\|^{2}\right)^{\prime}$ and that 'Simulation $E\left(\left\|\mathbf{x}^{\prime(k=1)}-\mathbf{x}\right\|^{2}\right)^{\prime}$ is approximately equal to 'Simulation $E\left(\left\|\mathbf{x}^{\prime(k=1, l=1)}-\mathbf{x}\right\|^{2}\right)^{\prime}$.
It should be noted that 'Simulation $E\left(\left\|\mathbf{x}^{\prime(k=1, l=1)}-\mathbf{x}\right\|^{2}\right)^{\prime}$ and 'Analytic $E\left(\left\|\mathbf{x}^{(k=1, l=1)}-\mathbf{x}\right\|^{2}\right)^{\prime}$ show excellent agreements, which validates (18) with $k=1$. Therefore, we can use analytically derived expression 'Analytic $E\left(\| \mathbf{x}^{\prime(k=1, l=1)}\right.$ $\left.-\mathbf{x} \|^{2}\right)^{\prime}$ to see how 'Simulation $E\left(\left\|\mathbf{x}^{\prime}-\mathbf{x}\right\|^{2}\right)^{\prime}$.

The same observations can be made in Figure 5(b). The results in Figure 5(b) with 'Simulation $E\left(\left\|\mathbf{x}^{\prime}-\mathbf{x}\right\|^{2}\right)^{\prime}$, 'Simulation $E\left(\left\|\mathbf{x}^{\prime(k=2)}-\mathbf{x}\right\|^{2}\right)^{\prime}$, 'Simulation $E\left(\| \mathbf{x}^{(k=2, l=1)}-\right.$ $\left.\mathbf{x} \|^{2}\right)^{\prime}$, and 'Analytic $E\left(\left\|\mathbf{x}^{(k=2, l=1)}-\mathbf{x}\right\|^{2}\right)^{\prime}$ are obtained from (31), (34), (35), and (27), respectively. Note that the results in Figure 5(b) are for the second-order $K$-approximation and that the results in Figure 5(c) are for the third-order $K$ approximation. The results in Figure 5(b) with 'Simulation 


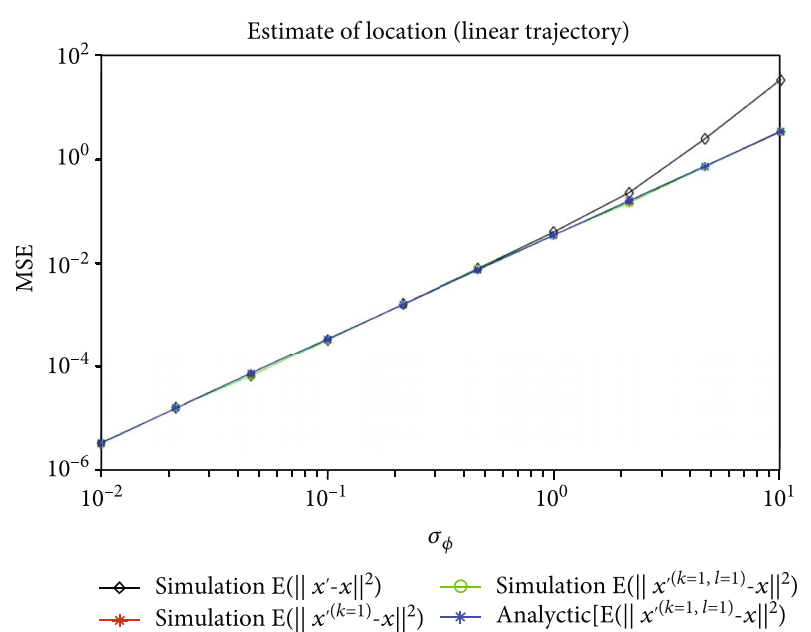

(a)

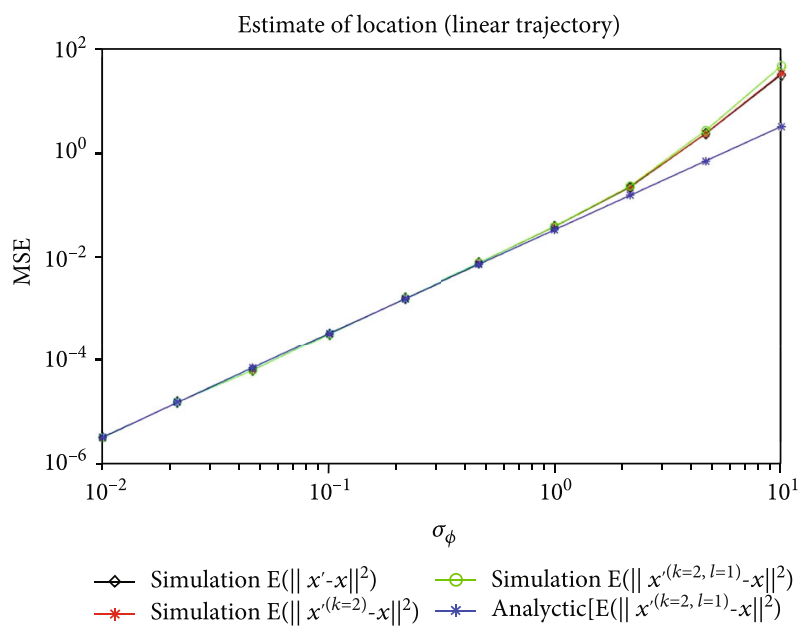

(b)

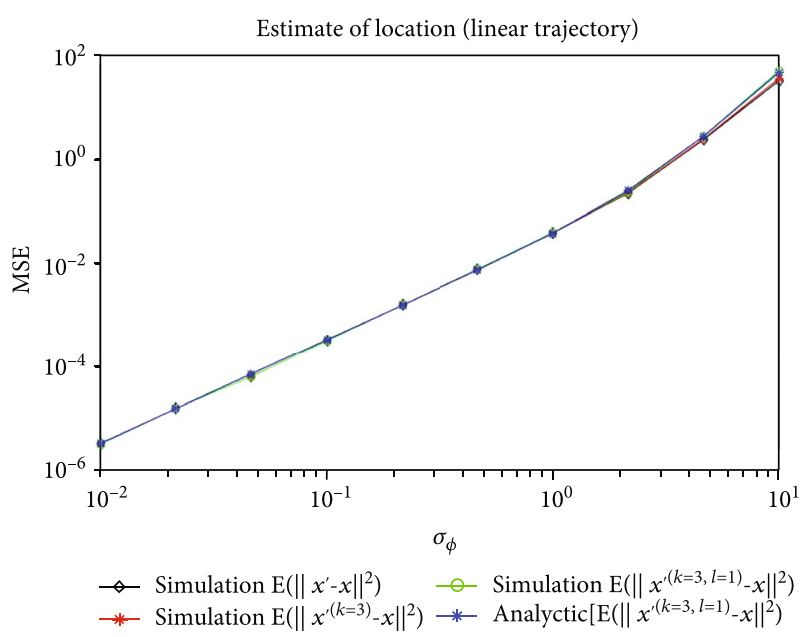

(c)

FIGURE 5: MSE of target location estimate for the linear trajectory: (a) first-order $K$-approximation, (b) second-order $K$-approximation, and (c) third-order $K$-approximation.

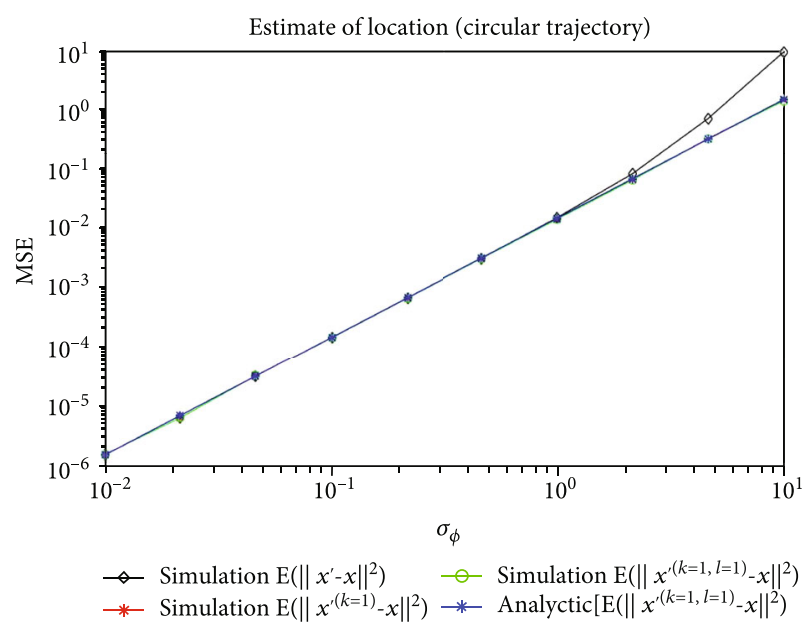

(a)

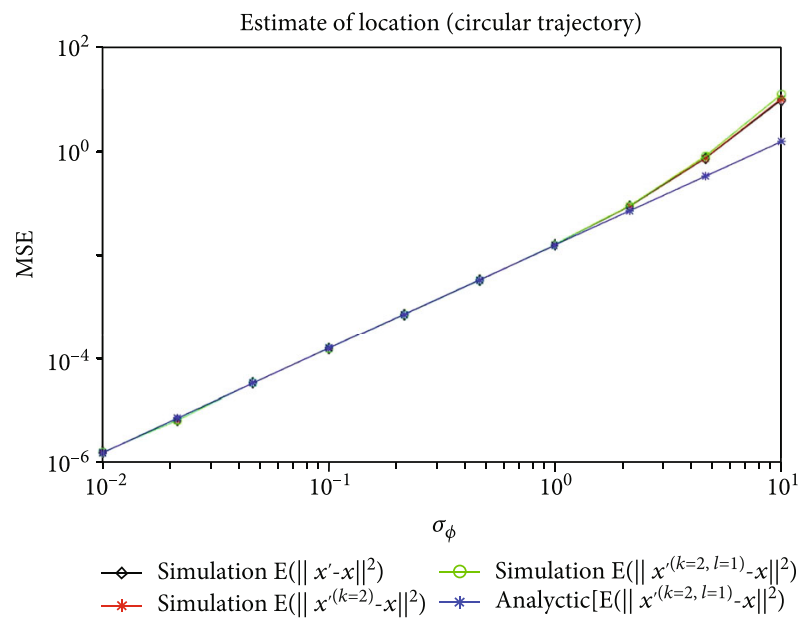

(b)

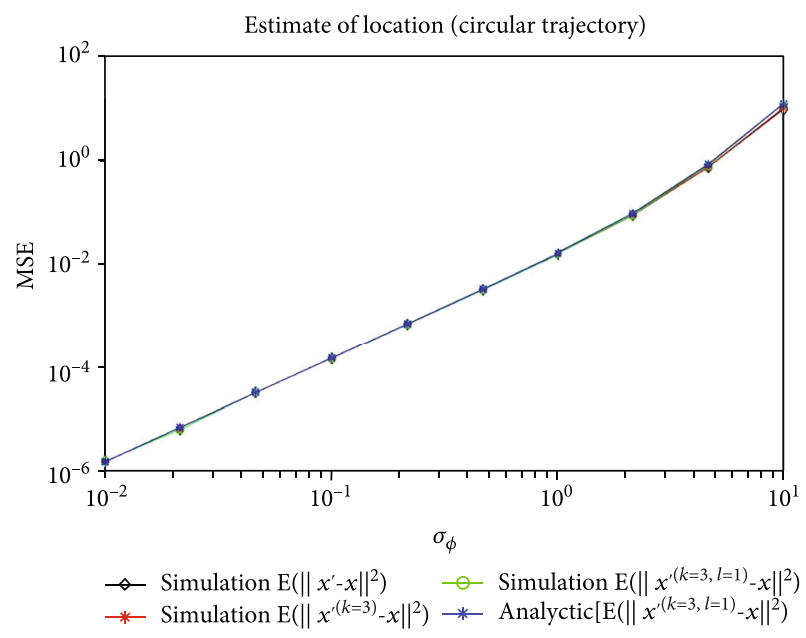

(c)

FIGURE 6: MSE of target location estimate for the circular trajectory: (a) first-order $K$-approximation, (b) second-order $K$-approximation, and (c) third-order $K$-approximation. 
$E\left(\left\|\mathbf{x}^{\prime}-\mathbf{x}\right\|^{2}\right)^{\prime}$, 'Simulation $E\left(\left\|\mathbf{x}^{\prime(k=3)}-\mathbf{x}\right\|^{2}\right)^{\prime}$, 'Simulation $E$ $\left(\left\|\mathbf{x}^{\prime(k=3, l=1)}-\mathbf{x}\right\|^{2}\right)^{\prime}$, and 'Analytic $E\left(\left\|\mathbf{x}^{\prime(k=3, l=1)}-\mathbf{x}\right\|^{2}\right)^{\prime}$ are obtained from (31), (36), (37), and (27), respectively.

To reduce the error due to $K$-approximation, the secondorder Taylor series can be used, which is illustrated in Figure 5(b). In Figure 5(a), difference between 'Simulation $E\left(\left\|\mathbf{x}^{\prime}-\mathbf{x}\right\|^{2}\right)^{\prime}$ and 'Simulation $E\left(\left\|\mathbf{x}^{\prime(k=1)}-\mathbf{x}\right\|^{2}\right)^{\prime}$ is much greater than that between 'Simulation $E\left(\left\|\mathbf{x}^{(k=1)}-\mathbf{x}\right\|^{2}\right)^{\prime}$ and 'Simulation $E\left(\left\|\mathbf{x}^{(k=1, l=1)}-\mathbf{x}\right\|^{2}\right)^{\prime}$ for large standard deviation of AOA estimation. On the other hand, difference between 'Simulation $E\left(\left\|\mathbf{x}^{\prime}-\mathbf{x}\right\|^{2}\right)^{\prime}$ and 'Simulation $E$ $\left(\left\|\mathbf{x}^{\prime(k=1)}-\mathbf{x}\right\|^{2}\right)^{\prime}$ is much smaller than that between Simulation $E\left(\left\|\mathbf{x}^{\prime(k=1)}-\mathbf{x}\right\|^{2}\right)^{\prime}$ and 'Simulation $E\left(\| \mathbf{x}^{\prime(k=1, l=1)}\right.$ $\left.-\mathbf{x} \|^{2}\right)^{\prime}$ for large standard deviation of AOA estimation in Figure 5(c). Actually, 'Simulation $E\left(\left\|\mathbf{x}^{\prime}-\mathbf{x}\right\|^{2}\right)^{\prime}$ and ' Simulation $E\left(\left\|\mathbf{x}^{\prime(k=3)}-\mathbf{x}\right\|^{2}\right)^{\prime}$ in Figure $5(\mathrm{c})$ is approximately equal for all standard deviation of AOA estimation error.

In Figure 6(a), it is clear that the difference between 'Simulation $E\left(\left\|\mathbf{x}^{\prime}-\mathbf{x}\right\|^{2}\right)^{\prime} \quad$ and 'Simulation $E\left(\| \mathbf{x}^{\prime(k=1)}-\right.$ $\left.\mathbf{x} \|^{2}\right)^{\prime}$ gets larger with the increase of standard deviation of AOA estimation, which implies that the error due to the first-order $K$-approximation becomes greater with the increase of standard deviation of AOA. 'Simulation $E$ $\left(\left\|\mathbf{x}^{\prime}-\mathbf{x}\right\|^{2}\right)^{\prime}$ and 'Simulation $E\left(\left\|\mathbf{x}^{\prime(k=1)}-\mathbf{x}\right\|^{2}\right)^{\prime}$ are approximately equal for standard deviation of $\sigma_{\phi}=0.2154^{\circ}$, which implies that error due to $K$-approximation is very small for standard deviation of $\sigma_{\phi}=0.2154^{\circ}$. The difference between 'Simulation $E\left(\left\|\mathbf{x}^{\prime(k=1)}-\mathbf{x}\right\|^{2}\right)^{\prime}$ and 'Simulation $E\left(\| \mathbf{x}^{\prime(k=1, l=1)}\right.$ $\left.-\mathbf{x} \|^{2}\right)^{\prime}$ quantifies how much error occurs due to $L$-approximation. The difference between 'Simulation $E\left(\| \mathbf{x}^{\prime(k=1)}-\right.$ $\left.\mathbf{x} \|^{2}\right)^{\prime}$ and 'Simulation $E\left(\left\|\mathbf{x}^{\prime(k=1, l=1)}-\mathbf{x}\right\|^{2}\right)^{\prime}$ is negligible for standard deviation of $\sigma_{\phi}=0.2154^{\circ}$, which means that $L$ -approximation results in approximately no error for standard deviation of $\sigma_{\phi}=0.2154^{\circ}$. Comparing 'Simulation $E$ $\left(\left\|\mathbf{x}^{\prime}-\mathbf{x}\right\|^{2}\right)^{\prime}, \quad$ 'Simulation $E\left(\left\|\mathbf{x}^{\prime(k=1)}-\mathbf{x}\right\|^{2}\right)^{\prime}, \quad$ and Simulation $E\left(\left\|\mathbf{x}^{\prime(k=1, l=1)}-\mathbf{x}\right\|^{2}\right)^{\prime}$, the first-order $K$-approximation induces much larger error than $L$-approximation.

The error due to $K$-approximation can be reduced by adopting the second-order Taylor series in the $K$-approximation, which is illustrated in Figure 6(b). Compared with the results in Figure 6(b), the difference between ' Simulation $E\left(\left\|\mathbf{x}^{\prime}-\mathbf{x}\right\|^{2}\right)^{\prime}$ and 'Simulation $E\left(\left\|\mathbf{x}^{\prime(k=2)}-\mathbf{x}\right\|^{2}\right)$ ' for the second-order $K$-approximation is smaller than that between 'Simulation $E\left(\left\|\mathbf{x}^{\prime}-\mathbf{x}\right\|^{2}\right)^{\prime}$ and 'Simulation $E$ $\left(\left\|\mathbf{x}^{\prime(k=1)}-\mathbf{x}\right\|^{2}\right)^{\prime}$ for the first-order $K$-approximation.
The error due to $K$-approximation can be made smaller by adopting the second-order $K$-approximation, which is illustrated in Figure 6(c). In Figure 6(c), it is shown that ' Simulation $E\left(\left\|\mathbf{x}^{\prime}-\mathbf{x}\right\|^{2}\right)^{\prime}$ and 'Simulation $E\left(\left\|\mathbf{x}^{\prime(k=3)}-\mathbf{x}\right\|^{2}\right)$ ' show agreement for all standard deviation of AOA estimation, which is quite different from 'Simulation $E\left(\left\|\mathbf{x}^{\prime}-\mathbf{x}\right\|^{2}\right)^{\prime}$ and 'Simulation $E\left(\left\|\mathbf{x}^{\prime(k=1)}-\mathbf{x}\right\|^{2}\right)^{\prime}$ in Figure 6(a).

\section{Conclusion}

Performance analysis of AOA-based localization is considered in this paper. Monte Carlo-based performance analysis is computationally very intensive, especially for a large number of repetitions. Closed-form expression of the meansquared error (MSE) of location estimate has been derived, and the validity is shown in the numerical results. The usefulness of the derivation lies in the fact that the MSE can be analytically obtained in a closed-form without computationally intensive Monte Carlo simulation. Since error due to $K$ -approximation and $L$-approximation is highly dependent on the noise variance, the scheme is more useful for high SNR. To reduce the error due to $K$-approximation and $L$ -approximation, higher order Taylor series can be adopted to reduce error at the expense of more computation in calculating the analytic MSE for taking higher order terms.

\section{Appendix}

\section{A. Second-Order Approximation of $\mathbf{A}^{\prime H} \mathbf{A}^{\prime}$ and $\mathbf{A}^{\prime H} \mathbf{b}^{\prime}$}

The second-order Taylor expansion-based approximation of the entries of $\mathbf{A}^{\prime H} \mathbf{A}^{\prime}$ and $\mathbf{A}^{\prime H} \mathbf{b}^{\prime}$ is presented.

$$
\begin{aligned}
\left(\mathbf{A}^{\prime H} \mathbf{A}^{\prime}\right)_{11}= & \sum_{i=1}^{N} \sin ^{2}\left(\phi_{i}+\delta \phi_{i}\right)=\sum_{i=1}^{N} \frac{1}{2}\left(1-\cos \left(2 \phi_{i}+2 \delta \phi_{i}\right)\right) \\
= & \sum_{i=1}^{N} \frac{1}{2}\left(1-\cos 2 \phi_{i} \cos 2 \delta \phi_{i}+\sin 2 \phi_{i} \sin 2 \delta \phi_{i}\right) \\
& \cdot\left(\cos 2 \delta \phi_{i} \simeq 1-\frac{\left(2 \delta \phi_{i}\right)^{2}}{2}, \sin 2 \delta \phi_{i} \simeq 2 \delta \phi_{i}\right) \\
\simeq & \sum_{i=1}^{N} \frac{1}{2}\left(1-\cos 2 \phi_{i}+2 \delta \phi_{i}^{2} \cos 2 \phi_{i}+2 \delta \phi_{i} \sin 2 \phi_{i}\right) \\
= & \sum_{i=1}^{N} \sin ^{2} \phi_{i}+\sum_{i=1}^{N}\left(\delta \phi_{i}^{2} \cos 2 \phi_{i}+\delta \phi_{i} \sin 2 \phi_{i}\right) \\
\equiv & \left(\mathbf{A}^{H} \mathbf{A}+\delta\left(\mathbf{A}^{H} \mathbf{A}\right)^{(k=2)}\right)_{11}, \\
\left(\mathbf{A}^{H} \mathbf{A}\right)_{11}= & \sum_{i=1}^{N}\left(\sin ^{2} \phi_{i}\right) \delta, \\
\left(\mathbf{A}^{H} \mathbf{A}\right)_{11}^{(k=2)}= & \sum_{i=1}^{N}\left(\delta \phi_{i}^{2} \cos 2 \phi_{i}+\delta \phi_{i} \sin 2 \phi_{i}\right),
\end{aligned}
$$




$$
\begin{aligned}
& \left(\mathbf{A}^{\prime H} \mathbf{A}^{\prime}\right)_{12}=\left(\mathbf{A}^{\prime H} \mathbf{A}^{\prime}\right)_{21}=-\frac{1}{2} \sum_{i=1}^{N} \sin 2\left(\phi_{i}+\delta \phi_{i}\right) \\
& =\sum_{i=1}^{N}-\frac{1}{2}\left(\sin 2 \phi_{i} \cos 2 \delta \phi_{i}+\cos 2 \phi_{i} \sin 2 \delta \phi_{i}\right) \\
& \cdot\left(\cos 2 \delta \phi_{i} \simeq 1-\frac{\left(2 \delta \phi_{i}\right)^{2}}{2}, \sin 2 \delta \phi_{i} \simeq 2 \delta \phi_{i}\right) \\
& \simeq \sum_{i=1}^{N}-\frac{1}{2}\left(\sin 2 \phi_{i}-2 \delta \phi_{i}^{2} \sin 2 \phi_{i}+2 \delta \phi_{i} \cos 2 \phi_{i}\right) \\
& =\sum_{i=1}^{N}-\frac{1}{2} \sin 2 \phi_{i}+\sum_{i=1}^{N}\left(\delta \phi_{i}^{2} \sin 2 \phi_{i}-\delta \phi_{i} \cos 2 \phi_{i}\right) \\
& \equiv\left(\mathbf{A}^{H} \mathbf{A}+\delta\left(\mathbf{A}^{H} \mathbf{A}\right)^{(k=2)}\right)_{12} \text {, } \\
& \left(\mathbf{A}^{H} \mathbf{A}\right)_{12}=\sum_{i=1}^{N}\left(-\frac{1}{2} \sin 2 \phi_{i}\right), \\
& \delta\left(\mathbf{A}^{H} \mathbf{A}\right)_{12}^{(k=2)}=\sum_{i=1}^{N}\left(\delta \phi_{i}^{2} \sin 2 \phi_{i}-\delta \phi_{i} \cos 2 \phi_{i}\right), \\
& \left(\mathbf{A}^{\prime H} \mathbf{A}^{\prime}\right)_{22}=\sum_{i=1}^{N} \cos ^{2}\left(\phi_{i}+\delta \phi_{i}\right)=\sum_{i=1}^{N} \frac{1}{2}\left(1+\cos \left(2 \phi_{i}+2 \delta \phi_{i}\right)\right) \\
& =\sum_{i=1}^{N} \frac{1}{2}\left(1+\cos 2 \phi_{i} \cos 2 \delta \phi_{i}-\sin 2 \phi_{i} \sin 2 \delta \phi_{i}\right) \\
& \text { - }\left(\cos 2 \delta \phi_{i} \simeq 1-\frac{\left(2 \delta \phi_{i}\right)^{2}}{2}, \sin 2 \delta \phi_{i} \simeq 2 \delta \phi_{i}\right) \\
& \simeq \sum_{i=1}^{N} \frac{1}{2}\left(1+\cos 2 \phi_{i}-2 \delta \phi_{i}^{2} \cos 2 \phi_{i}-2 \delta \phi_{i} \sin 2 \phi_{i}\right) \\
& =\sum_{i=1}^{N} \cos ^{2} \phi_{i}+\sum_{i=1}^{N}\left(-\delta \phi_{i}^{2} \cos 2 \phi_{i}-\delta \phi_{i} \sin 2 \phi_{i}\right) \\
& \equiv\left(\mathbf{A}^{H} \mathbf{A}+\delta\left(\mathbf{A}^{H} \mathbf{A}\right)^{(k=2)}\right)_{22} \text {, } \\
& \left(\mathbf{A}^{H} \mathbf{A}\right)_{22}=\sum_{i=1}^{N}\left(\cos ^{2} \phi_{i}\right), \\
& \delta\left(\mathbf{A}^{H} \mathbf{A}\right)_{22}^{(k=2)}=\sum_{i=1}^{N}\left(-\delta \phi_{i}^{2} \cos 2 \phi_{i}-\delta \phi_{i} \sin 2 \phi_{i}\right),
\end{aligned}
$$

$$
\begin{aligned}
\left(\mathbf{A}^{\prime H} \mathbf{b}^{\prime}\right)_{1} & =\sum_{i=1}^{N}\left(x_{i} \sin ^{2}\left(\phi_{i}+\delta \phi_{i}\right)-\frac{y_{i}}{2} \sin 2\left(\phi_{i}+\delta \phi_{i}\right)\right) \\
\simeq & \sum_{i=1}^{N}\left[\left(\frac{x_{i}}{2}\left(1-\left(\cos 2 \phi_{i} \cos 2 \delta \phi_{i}-\sin 2 \phi_{i} \sin 2 \delta \phi_{i}\right)\right)\right)\right. \\
& -\frac{y_{i}}{2}\left(\sin 2 \phi_{i}-2 \delta \phi_{i}^{2} \sin 2 \phi_{i}+2 \delta \phi_{i} \cos 2 \phi_{i}\right)
\end{aligned}
$$

$$
\begin{aligned}
& =\sum_{i=1}^{N}\left(x_{i} \sin ^{2} \phi_{i}-\frac{1}{2} y_{i} \sin 2 \phi_{i}\right) \\
& +\sum_{i=1}^{N}\left(\delta \phi_{i}^{2}\left(x_{i} \cos 2 \phi_{i}+y_{i} \sin 2 \phi_{i}\right)\right. \\
& \left.+\delta \phi_{i}\left(x_{i} \sin 2 \phi_{i}-y_{i} \cos 2 \phi_{i}\right)\right) \equiv\left(\mathbf{A}^{H} \mathbf{b}+\delta\left(\mathbf{A}^{H} \mathbf{b}\right)^{(k=2)}\right)_{1}, \\
& \left(\mathbf{A}^{H} \mathbf{b}\right)_{1}=\sum_{i=1}^{N}\left(x_{i} \sin ^{2} \phi_{i}+\frac{1}{2} y_{i} \sin 2 \phi_{i}\right), \\
& \delta\left(\mathbf{A}^{H} \mathbf{b}\right)_{1}^{(k=2)}=\sum_{i=1}^{N}\left(\begin{array}{c}
\delta \phi_{i}^{2}\left(x_{i} \cos 2 \phi_{i}+y_{i} \sin 2 \phi_{i}\right) \\
+\delta \phi_{i}\left(x_{i} \sin 2 \phi_{i}-y_{i} \cos 2 \phi_{i}\right)
\end{array}\right) \text {, } \\
& \left(\mathbf{A}^{\prime H} \mathbf{b}^{\prime}\right)_{2}=\sum_{i=1}^{N}\left(-\frac{x_{i}}{2} \sin 2\left(\phi_{i}+\delta \phi_{i}\right)+y_{i} \cos ^{2}\left(\phi_{i}+\delta \phi_{i}\right)\right) \\
& \simeq \sum_{i=1}^{N}\left(-\frac{x_{i}}{2}\left(\sin 2 \phi_{i}-2 \delta \phi_{i}^{2} \sin 2 \phi_{i}+2 \delta \phi_{i} \cos 2 \phi_{i}\right)\right. \\
& \left.+\frac{y_{i}}{2}\left(1+\cos 2 \phi_{i}-2 \delta \phi_{i}^{2} \cos 2 \phi_{i}-2 \delta \phi_{i} \sin 2 \phi_{i}\right)\right) \\
& =\sum_{i=1}^{N}-\left(\frac{x_{i}}{2} \sin 2 \phi_{i}-y_{i} \cos ^{2} \phi_{i}\right) \\
& -\sum_{i=1}^{N}\left(\delta \phi_{i}\left(x_{i} \cos 2 \phi_{i}+y_{i} \sin 2 \phi_{i}\right)\right. \\
& \left.-\delta \phi_{i}^{2}\left(x_{i} \sin 2 \phi_{i}-y_{i} \cos 2 \phi_{i}\right)\right) \\
& \equiv\left(\mathbf{A}^{H} \mathbf{b}+\delta\left(\mathbf{A}^{H} \mathbf{b}\right)^{(k=2)}\right)_{2} \text {, } \\
& \left(\mathbf{A}^{H} \mathbf{b}\right)_{2}=\sum_{i=1}^{N}-\left(\frac{1}{2} x_{i} \sin 2 \phi_{i}+y_{i} \cos ^{2} \phi_{i}\right), \\
& \delta\left(\mathbf{A}^{H} \mathbf{b}\right)_{2}^{(k=2)}=-\sum_{i=1}^{N}\left(\begin{array}{c}
\delta \phi_{i}\left(x_{i} \cos 2 \phi_{i}+y_{i} \sin 2 \phi_{i}\right) \\
-\delta \phi_{i}^{2}\left(x_{i} \sin 2 \phi_{i}-y_{i} \cos 2 \phi_{i}\right)
\end{array}\right) .
\end{aligned}
$$

\section{B. Third-Order Approximation of $\mathbf{A}^{\prime H} \mathbf{A}^{\prime}$ and} $\mathbf{A}^{\prime H} \mathbf{b}^{\prime}$

The third-order Taylor expansion-based approximation of the entries of $\mathbf{A}^{\prime H} \mathbf{A}^{\prime}$ and $\mathbf{A}^{\prime H} \mathbf{b}^{\prime}$ is presented.

$$
\begin{aligned}
& \left(\mathbf{A}^{\prime H} \mathbf{A}^{\prime}\right)_{11} \\
& =\sum_{i=1}^{N} \sin ^{2}\left(\phi_{i}+\delta \phi_{i}\right)=\sum_{i=1}^{N} \frac{1}{2}\left(1-\cos \left(2 \phi_{i}+2 \delta \phi_{i}\right)\right) \\
& =\sum_{i=1}^{N} \frac{1}{2}\left(1-\cos 2 \phi_{i} \cos 2 \delta \phi_{i}+\sin 2 \phi_{i} \sin 2 \delta \phi_{i}\right)
\end{aligned}
$$


$\cdot\left(\cos 2 \delta \phi_{i} \simeq 1-\frac{\left(2 \delta \phi_{i}\right)^{2}}{2}, \sin 2 \delta \phi_{i} \simeq 2 \delta \phi_{i}-\frac{\left(2 \delta \phi_{i}\right)^{3}}{6}\right)$

$\simeq \sum_{i=1}^{N} \frac{1}{2}\left(1-\cos 2 \phi_{i}+2 \delta \phi_{i}^{2} \cos 2 \phi_{i}+2 \delta \phi_{i} \sin 2 \phi_{i}\right.$

$\left.-\frac{4}{3} \delta \phi_{i}^{3} \sin 2 \phi_{i}\right)=\sum_{i=1}^{N} \sin ^{2} \phi_{i}+\sum_{i=1}^{N}\left(-\frac{2}{3} \delta \phi_{i}^{3} \sin 2 \phi_{i}\right.$

$\left.+\delta \phi_{i}^{2} \cos 2 \phi_{i}+\delta \phi_{i} \sin 2 \phi_{i}\right) \equiv\left(\mathbf{A}^{H} \mathbf{A}+\delta\left(\mathbf{A}^{H} \mathbf{A}\right)^{(k=2)}\right)_{11}$,

$\left(\mathbf{A}^{H} \mathbf{A}\right)_{11}=\sum_{i=1}^{N}\left(\sin ^{2} \phi_{i}\right)$

$\delta\left(\mathbf{A}^{H} \mathbf{A}\right)_{11}^{(k=3)}=\sum_{i=1}^{N}\left(-\frac{2}{3} \delta \phi_{i}^{3} \sin 2 \phi_{i}+\delta \phi_{i}^{2} \cos 2 \phi_{i}\right.$

$\left.+\delta \phi_{i} \sin 2 \phi_{i}\right)$

$$
\begin{aligned}
\left(\mathbf{A}^{\prime H} \mathbf{A}^{\prime}\right)_{12} & \left(\mathbf{A}^{\prime H} \mathbf{A}^{\prime}\right)_{21}=-\frac{1}{2} \sum_{i=1}^{N} \sin 2\left(\phi_{i}+\delta \phi_{i}\right) \\
= & \sum_{i=1}^{N}-\frac{1}{2}\left(\sin 2 \phi_{i} \cos 2 \delta \phi_{i}+\cos 2 \phi_{i} \sin 2 \delta \phi_{i}\right) \\
& \cdot\left(\cos 2 \delta \phi_{i} \simeq 1-\frac{\left(2 \delta \phi_{i}\right)^{2}}{2}, \sin 2 \delta \phi_{i} \simeq 2 \delta \phi_{i}-\frac{\left(2 \delta \phi_{i}\right)^{3}}{6}\right) \\
\simeq & \sum_{i=1}^{N}-\frac{1}{2}\left(\sin 2 \phi_{i}-2 \delta \phi_{i}^{2} \sin 2 \phi_{i}+2 \delta \phi_{i} \cos 2 \phi_{i}\right. \\
& \left.-\frac{4}{3} \delta \phi_{i}^{3} \cos 2 \phi_{i}\right)=\sum_{i=1}^{N}-\frac{1}{2} \sin 2 \phi_{i} \\
& +\sum_{i=1}^{N}\left(\frac{2}{3} \delta \phi_{i}^{3} \cos 2 \phi_{i}+\delta \phi_{i}^{2} \sin 2 \phi_{i}-\delta \phi_{i} \cos 2 \phi_{i}\right) \\
\equiv & \left(\mathbf{A}^{H} \mathbf{A}+\delta\left(\mathbf{A}^{H} \mathbf{A}\right)^{(k=3)}\right)_{12}, \\
& \quad\left(\mathbf{A}^{H} \mathbf{A}\right)_{12}=\sum_{i=1}^{N}\left(-\frac{1}{2} \sin 2 \phi_{i}\right),
\end{aligned}
$$$$
\delta\left(\mathbf{A}^{H} \mathbf{A}\right)_{12}^{(k=3)}=\sum_{i=1}^{N}\left(\frac{2}{3} \delta \phi_{i}^{3} \cos 2 \phi_{i}+\delta \phi_{i}^{2} \sin 2 \phi_{i}-\delta \phi_{i} \cos 2 \phi_{i}\right),
$$

$$
\begin{aligned}
\left(\mathbf{A}^{\prime H} \mathbf{A}^{\prime}\right)_{22} & \\
= & \sum_{i=1}^{N} \cos ^{2}\left(\phi_{i}+\delta \phi_{i}\right)=\sum_{i=1}^{N} \frac{1}{2}\left(1+\cos \left(2 \phi_{i}+2 \delta \phi_{i}\right)\right) \\
= & \sum_{i=1}^{N} \frac{1}{2}\left(1+\cos 2 \phi_{i} \cos 2 \delta \phi_{i}-\sin 2 \phi_{i} \sin 2 \delta \phi_{i}\right) \\
& \cdot\left(\cos 2 \delta \phi_{i} \simeq 1-\frac{\left(2 \delta \phi_{i}\right)^{2}}{2}, \sin 2 \delta \phi_{i} \simeq 2 \delta \phi_{i}-\frac{\left(2 \delta \phi_{i}\right)^{3}}{6}\right) \\
\simeq & \sum_{i=1}^{N} \frac{1}{2}\left(1+\cos 2 \phi_{i}-2 \delta \phi_{i}^{2} \cos 2 \phi_{i}-2 \delta \phi_{i} \sin 2 \phi_{i}\right. \\
& \left.+\frac{8}{6} \delta \phi_{i}^{3} \sin 2 \phi_{i}\right)=\sum_{i=1}^{N} \cos \phi_{i}+\sum_{i=1}^{N}\left(-\delta \phi_{i}^{2} \cos 2 \phi_{i}\right. \\
& \left.-\delta \phi_{i} \sin 2 \phi_{i}+\frac{2}{3} \delta \phi_{i}^{3} \sin 2 \phi_{i}\right) \equiv\left(\mathbf{A}^{H} \mathbf{A}+\delta\left(\mathbf{A}^{H} \mathbf{A}\right)^{(k=3)}\right)_{22}, \\
& \left(\mathbf{A}^{H} \mathbf{A}\right)_{22}=\sum_{i=1}^{N}\left(\cos ^{2} \phi_{i}\right), \\
\delta( & \left.\mathbf{A}^{H} \mathbf{A}\right)_{22}^{(k=3)}=\sum_{i=1}^{N}\left(\frac{2}{3} \delta \phi_{i}^{3} \sin 2 \phi_{i}-\delta \phi_{i}^{2} \cos 2 \phi_{i}-\delta \phi_{i} \sin 2 \phi_{i}\right),
\end{aligned}
$$

$$
\begin{aligned}
&\left(\mathbf{A}^{\prime H} \mathbf{b}^{\prime}\right)_{1} \\
&= \sum_{i=1}^{N}\left(x_{i} \sin ^{2}\left(\phi_{i}+\delta \phi_{i}\right)-\frac{y_{i}}{2} \sin 2\left(\phi_{i}+\delta \phi_{i}\right)\right) \\
& \simeq \sum_{i=1}^{N}\left[\left(\frac{x_{i}}{2}\left(1-\left(\cos 2 \phi_{i} \cos 2 \delta \phi_{i}-\sin 2 \phi_{i} \sin 2 \delta \phi_{i}\right)\right)\right)\right. \\
&-\frac{y_{i}}{2}\left(\sin 2 \phi_{i}-2 \delta \phi_{i}^{2} \sin 2 \phi_{i}+2 \delta \phi_{i} \cos 2 \phi_{i}\right. \\
&\left.\left.-\frac{4}{3} \delta \phi_{i}^{3} \cos 2 \phi_{i}\right)\right]=\sum_{i=1}^{N}\left(x_{i} \sin ^{2} \phi_{i}-\frac{1}{2} y_{i} \sin 2 \phi_{i}\right) \\
&-\sum_{i=1}^{N}\left(\frac{2}{3} \delta \phi_{i}^{3}\left(x_{i} \sin 2 \phi_{i}-y_{i} \cos 2 \phi_{i}\right)-\delta \phi_{i}^{2}\left(x_{i} \cos 2 \phi_{i}\right.\right. \\
&\left.\left.+y_{i} \sin 2 \phi_{i}\right)-\delta \phi_{i}\left(x_{i} \sin 2 \phi_{i}-y_{i} \cos 2 \phi_{i}\right)\right) \\
& \equiv\left(\mathbf{A}^{H} \mathbf{b}+\delta\left(\mathbf{A}^{H} \mathbf{b}\right)^{(k=3)}\right)_{1}, \\
&\left(\mathbf{A}^{H} \mathbf{b}\right)_{1}=\sum_{i=1}^{N}\left(x_{i} \sin ^{2} \phi_{i}+\frac{1}{2} y_{i} \sin 2 \phi_{i}\right), \\
& \delta\left(\mathbf{A}^{H} \mathbf{b}\right)_{1}^{(k=3)}=-\sum_{i=1}^{N}\left(\begin{array}{l}
\frac{2}{3} \delta \phi_{i}^{3}\left(x_{i} \sin 2 \phi_{i}-y_{i} \cos 2 \phi_{i}\right) \\
-\delta \phi_{i}^{2}\left(x_{i} \cos 2 \phi_{i}+y_{i} \sin 2 \phi_{i}\right) \\
-\delta \phi_{i}\left(x_{i} \sin 2 \phi_{i}-y_{i} \cos 2 \phi_{i}\right)
\end{array}\right),
\end{aligned}
$$




$$
\begin{aligned}
& \left(\mathbf{A}^{\prime H} \mathbf{b}^{\prime}\right)_{2} \\
& =\sum_{i=1}^{N}\left(-\frac{x_{i}}{2} \sin 2\left(\phi_{i}+\delta \phi_{i}\right)+y_{i} \cos ^{2}\left(\phi_{i}+\delta \phi_{i}\right)\right) \\
& \simeq \sum_{i=1}^{N}\left(-\frac{x_{i}}{2}\left(\sin 2 \phi_{i}-2 \delta \phi_{i}^{2} \sin 2 \phi_{i}+2 \delta \phi_{i} \cos 2 \phi_{i}\right.\right. \\
& \left.-\frac{4}{3} \delta \phi_{i}^{3} \cos 2 \phi_{i}\right)+\frac{y_{i}}{2}\left(1+\cos 2 \phi_{i}-2 \delta \phi_{i}^{2} \cos 2 \phi_{i}\right. \\
& \left.\left.-2 \delta \phi_{i} \sin 2 \phi_{i}+\frac{4}{3} \delta \phi_{i}^{3} \sin 2 \phi_{i}\right)\right)=\sum_{i=1}^{N} \\
& -\left(\frac{x_{i}}{2} \sin 2 \phi_{i}-y_{i} \cos ^{2} \phi_{i}\right)-\sum_{i=1}^{N}\left(-\frac{2}{3} \delta \phi_{i}^{3}\left(x_{i} \cos 2 \phi_{i}\right.\right. \\
& \left.+y_{i} \sin 2 \phi_{i}\right)+\delta \phi_{i}\left(x_{i} \cos 2 \phi_{i}+y_{i} \sin 2 \phi_{i}\right) \\
& \left.-\delta \phi_{i}^{2}\left(x_{i} \sin 2 \phi_{i}-y_{i} \cos 2 \phi_{i}\right)\right) \\
& \equiv\left(\mathbf{A}^{H} \mathbf{b}+\delta\left(\mathbf{A}^{H} \mathbf{b}\right)^{(k=3)}\right)_{2} \\
& \left(\mathbf{A}^{H} \mathbf{b}\right)_{2}=\sum_{i=1}^{N}\left(-\frac{1}{2} x_{i} \sin 2 \phi_{i}+y_{i} \cos ^{2} \phi_{i}\right) \text {, } \\
& \delta\left(\mathbf{A}^{H} \mathbf{b}\right)_{2}^{(k=3)}=-\sum_{i=1}^{N}\left(\begin{array}{c}
-\frac{2}{3} \delta \phi_{i}^{3}\left(x_{i} \cos 2 \phi_{i}+y_{i} \sin 2 \phi_{i}\right) \\
+\delta \phi_{i}\left(x_{i} \cos 2 \phi_{i}+y_{i} \sin 2 \phi_{i}\right) \\
-\delta \phi_{i}^{2}\left(x_{i} \sin 2 \phi_{i}-y_{i} \cos 2 \phi_{i}\right)
\end{array}\right) .
\end{aligned}
$$

\section{The Perturbation of the Solution of Linear System, $L$-Approximation of $\mathbf{x}^{\prime(k=1)}$}

Let

$$
\mathbf{C x}=\mathbf{G}
$$

denote an unperturbed linear system with $\mathbf{C} \in \mathbb{R}^{n \times n}, \mathbf{G} \in \mathbb{R}^{n}$ and $\mathbf{x} \in \mathbb{R}^{n}$.

Consider a perturbed linear system,

$$
(\mathbf{C}+\varepsilon \mathbf{F}) \mathbf{x}(\varepsilon)=\mathbf{G}+\varepsilon \mathbf{f} .
$$

where $\mathbf{F} \in \mathbb{R}^{n \times n}$ and $\mathbf{f} \in \mathbb{R}^{n}$. If $\mathbf{C}$ is nonsingular, then it is clear that $\mathbf{x}(\varepsilon)$ is differentiable in a neighborhood of zero.

Let the first derivative of $\mathbf{x}(\varepsilon)$ be denoted by $\dot{\mathbf{x}}(\varepsilon)$. Differentiation of (C.2) yields

$$
\mathbf{F x}(\varepsilon)+(\mathbf{C}+\varepsilon \mathbf{F}) \dot{\mathbf{x}}(\varepsilon)=\mathbf{f} .
$$

Substituting $\varepsilon=0$ in (C.3) yields

$$
\mathbf{F x}(\varepsilon=0)+\mathbf{C} \dot{\mathbf{x}}(\varepsilon=0)=\mathbf{f} .
$$

Comparing (C.1) and (C.2), it is clear that $\mathbf{x}(\varepsilon=0)$ in (C.4) is equal to $\mathbf{x}$ in (C.1):

$$
\mathbf{x} \equiv \mathbf{x}(\varepsilon=0)
$$

Using (C.5) in (C.4) results in

$$
\mathbf{F x}+\mathbf{C} \dot{\mathbf{x}}(\varepsilon=0)=\mathbf{f}
$$

Solving for $\dot{\mathbf{x}}(\varepsilon=0)$ yields

$$
\dot{\mathbf{x}}(\varepsilon=0)=\mathbf{C}^{-1}(f-\mathbf{F x}) .
$$

First-order approximation of $\mathbf{x}(\varepsilon)$ based on the Taylor expansion can be written as

$$
\mathbf{x}(\varepsilon) \approx \mathbf{x}(\varepsilon=0)+\varepsilon \dot{\mathbf{x}}(\varepsilon=0)=\mathbf{x}+\mathbf{C}^{-1}(\varepsilon f-\varepsilon \mathbf{F x}) .
$$

Comparing (C.2) and (16), $\varepsilon \mathbf{F}$ and $\varepsilon \mathbf{f}$ in (C.2) correspond to $\delta\left(\mathbf{A}^{H} \mathbf{A}\right)_{11}^{(k=1)}$ and $\delta\left(\mathbf{A}^{H} \mathbf{b}\right)_{11}^{(k=1)}$ in (16), respectively. Therefore, the approximation of $\mathbf{x}^{\prime(k)}$ and $\mathbf{x}^{\prime(k, l=1)}$, is obtained from (C.3) by substituting $\varepsilon \mathbf{f}=\delta\left(\mathbf{A}^{H} \mathbf{b}\right)^{(k)}$ and $\varepsilon \mathbf{F}=\delta\left(\mathbf{A}^{H} \mathbf{A}\right)^{(k)}$.

\section{Mean of Entries of $\delta\left(\mathbf{A}^{\prime H} \mathbf{A}^{\prime}\right)$ and $\delta\left(\mathbf{A}^{\prime H} \mathbf{b}^{\prime}\right)$ Based on the First-Order Taylor Series}

Explicit expression of many expectation terms in (28), (29) and (30) is derived. The expectation of $\delta \phi_{i} \delta \phi_{j}$ can be written as

$$
E\left(\delta \phi_{i} \delta \phi_{j}\right)= \begin{cases}\sigma_{\phi}^{2}, & i=j \\ 0, & \text { otherwise }\end{cases}
$$

Explicit expression of the expectation of $\left(\delta\left(\mathbf{A}^{H} \mathbf{A}\right)_{11}^{(k=1)}\right)^{2}$ is derived:

$$
\begin{aligned}
& E\left(\left(\delta\left(\mathbf{A}^{H} \mathbf{A}\right)_{11}^{(k=1)}\right)^{2}\right) \\
& =E\left(\left(\delta\left(\mathbf{A}^{H} \mathbf{A}\right)_{11}^{(k=1)}\right)^{2}\right)=E\left(\delta\left(\mathbf{A}^{H} \mathbf{A}\right)_{11}^{(k=1)} \delta\left(\mathbf{A}^{H} \mathbf{A}\right)_{11}^{(k=1)}\right) \\
& =E\left(\left(\sum_{i=1}^{N} \delta \phi_{i} \sin 2 \phi_{i}\left(\sum_{j=1}^{N} \delta \phi_{j} \sin 2 \phi_{j}\right)\right)\right) \\
& =E\left(\sum_{i=1}^{N} \delta \phi_{i}^{2}\right) \sum_{j=1}^{N} \sin ^{2} 2 \phi_{j}=\sigma_{\phi}^{2} \sum_{i=1}^{N} \sin ^{2} 2 \phi_{i} .
\end{aligned}
$$

Since the other entries can be derived similarly, we list the final expressions for the other entries:

$$
E\left(\left(\delta\left(\mathbf{A}^{H} \mathbf{A}\right)_{12}^{(k=1)}\right)^{2}\right)=\sigma_{\phi}^{2} \sum_{i=1}^{N} \cos ^{2} 2 \phi_{i},
$$




$$
\begin{aligned}
& E\left(\left(\delta\left(\mathbf{A}^{H} \mathbf{A}\right)_{22}^{(k=1)}\right)^{2}\right)=\sigma_{\phi}^{2} \sum_{i=1}^{N}\left(x_{i} \sin ^{2} 2 \phi_{i}\right) \\
& E\left(\delta\left(\mathbf{A}^{H} \mathbf{b}\right)_{11}^{(k=1)} \delta\left(\mathbf{A}^{H} \mathbf{A}\right)_{12}^{(k=1)}\right)=-\frac{1}{2} \sigma_{\phi}^{2} \sum_{i=1}^{N} \sin 4 \phi_{i} \\
& E\left(\delta\left(\mathbf{A}^{H} \mathbf{A}\right)_{11}^{(k=1)} \delta\left(\mathbf{A}^{H} \mathbf{A}\right)_{22}^{(k=1)}\right)=-\sigma_{\phi}^{2} \sum_{i=1}^{N} \sin ^{2} 2 \phi_{i} \\
& E\left(\left(\delta\left(\mathbf{A}^{H} \mathbf{b}\right)_{1}^{(k=1)}\right)^{2}\right) \\
& =\sigma_{\phi}^{2} \sum_{i=1}^{N}\left(x_{i}^{2} \sin ^{2} 2 \phi_{i}+y_{i}^{2} \cos ^{2} 2 \phi_{i}-x_{i} y_{i} \sin 4 \phi_{i}\right) \\
& E\left(\left(\delta\left(\mathbf{A}^{H} \mathbf{b}\right)_{2}^{(k=1)}\right)^{2}\right) \\
& =\sigma_{\phi}^{2} \sum_{i=1}^{N}\left(x_{i}^{2} \cos ^{2} 2 \phi_{i}+y_{i}^{2} \sin ^{2} 2 \phi_{i}+x_{i} y_{i} \sin 4 \phi_{i}\right) \\
& E\left(\delta\left(\mathbf{A}^{H} \mathbf{A}\right)_{11}^{(k=1)} \delta\left(\mathbf{A}^{H} \mathbf{b}\right)_{1}^{(k=1)}\right) \\
& =-\sigma_{\phi}^{2} \sum_{i=1}^{N}\left(-x_{i}^{2} \sin ^{2} 2 \phi_{i}+\frac{1}{2} y_{i} \sin 4 \phi_{i}\right) \\
& E\left(\delta\left(\mathbf{A}^{H} \mathbf{A}\right)_{11}^{(k=1)} \delta\left(\mathbf{A}^{H} \mathbf{b}\right)_{2}^{(k=1)}\right) \\
& =-\sigma_{\phi}^{2} \sum_{i=1}^{N}\left(\frac{1}{2} x_{i} \sin 4 \phi_{i}+y_{i} \sin ^{2} 2 \phi_{i}\right) \\
& E\left(\delta\left(\mathbf{A}^{H} \mathbf{A}\right)_{22}^{(k=1)} \delta\left(\mathbf{A}^{H} \mathbf{b}\right)_{1}^{(k=1)}\right) \\
& =-\sigma_{\phi}^{2} \sum_{i=1}^{N}\left(x_{i} \sin ^{2} 2 \phi_{i}-\frac{1}{2} y_{i} \sin 4 \phi_{i}\right) \\
& E\left(\delta\left(\mathbf{A}^{H} \mathbf{A}\right)_{22}^{(k=1)} \delta\left(\mathbf{A}^{H} \mathbf{b}\right)_{2}^{(k=1)}\right) \\
& =\sigma_{\phi}^{2} \sum_{i=1}^{N}\left(\frac{1}{2} x_{i} \sin 4 \phi_{i}+y_{i} \sin ^{2} 2 \phi_{i}\right) \\
& E\left(\delta\left(\mathbf{A}^{H} \mathbf{b}\right)_{1}^{(k=1)} \delta\left(\mathbf{A}^{H} \mathbf{A}\right)_{12}^{(k=1)}\right) \\
& =E\left(\delta\left(\mathbf{A}^{H} \mathbf{b}\right)_{1}^{(k=1)} \delta\left(\mathbf{A}^{H} \mathbf{A}\right)_{21}^{(k=1)}\right) \\
& =-\sigma_{\phi}^{2} \sum_{i=1}^{N}\left(\frac{1}{2} x_{i} \sin 4 \phi_{i}-y_{i} \cos ^{2} 2 \phi_{i}\right) \\
& E\left(\delta\left(\mathbf{A}^{H} \mathbf{b}\right)_{2}^{(k=1)} \delta\left(\mathbf{A}^{H} \mathbf{A}\right)_{12}^{(k=1)}\right) \\
& =E\left(\delta\left(\mathbf{A}^{H} \mathbf{b}\right)_{2}^{(k=1)} \delta\left(\mathbf{A}^{H} \mathbf{A}\right)_{21}^{(k=1)}\right) \\
& =\sigma_{\phi}^{2} \sum_{i=1}^{N}\left(x_{i} \cos ^{2} 2 \phi_{i}-\frac{1}{2} y_{i} \sin 4 \phi_{i}\right)
\end{aligned}
$$

$$
\begin{aligned}
E( & \left.\delta\left(\mathbf{A}^{H} \mathbf{b}\right)_{1}^{(k=1)} \delta\left(\mathbf{A}^{H} \mathbf{A}\right)_{2}^{(k=1)}\right) \\
& =\sigma_{\phi}^{2} \sum_{i=1}^{N}\left(\frac{1}{2}\left(x_{i}^{2}-y_{i}^{2}\right) \sin 4 \phi_{i}-x_{i} y_{i} \cos 4 \phi_{i}\right)
\end{aligned}
$$

E. Mean of Entries of $\delta\left(\mathbf{A}^{\prime H} \mathbf{A}^{\prime}\right)$ and $\delta\left(\mathbf{A}^{\prime}{ }^{\prime} \mathbf{b}^{\prime}\right)$ Based on the Second-Order Taylor Series

$$
\begin{aligned}
& E\left(\left(\delta\left(\mathbf{A}^{H} \mathbf{A}\right)_{11}^{(k=2)}\right)^{2}\right) \\
& =\sigma_{\phi}^{2} \sum_{i=1}^{N} \sin ^{2} 2 \phi_{i}+3 \sigma_{\phi}^{4} \sum_{i=1}^{N} \cos ^{2} 2 \phi_{i} \\
& +\sigma_{\phi}^{4} \sum_{i=1(i \neq j)}^{N} \sum_{j=1}^{N} \cos 2 \phi_{i} \cos 2 \phi_{j}, \\
& E\left(\left(\delta\left(\mathbf{A}^{H} \mathbf{A}\right)_{12}^{(k=2)}\right)^{2}\right) \\
& =\sigma_{\phi}^{2} \sum_{i=1}^{N} \cos ^{2} 2 \phi_{i}+3 \sigma_{\phi}^{4} \sum_{i=1}^{N} \sin ^{2} 2 \phi_{i} \\
& +\sigma_{\phi}^{4} \sum_{i=1(i \neq j)}^{N} \sum_{j=1}^{N} \sin 2 \phi_{i} \sin 2 \phi_{j}, \\
& E\left(\left(\delta\left(\mathbf{A}^{H} \mathbf{A}\right)_{22}^{(k=2)}\right)^{2}\right) \\
& =\sigma_{\phi}^{2} \sum_{i=1}^{N} \sin ^{2} 2 \phi_{i}+3 \sigma_{\phi}^{4} \sum_{i=1}^{N} \cos ^{2} 2 \phi_{i} \\
& +\sigma_{\phi}^{4} \sum_{i=1(i \neq j)}^{N} \sum_{j=1}^{N} \cos 2 \phi_{i} \cos 2 \phi_{j}, \\
& E\left(\left(\delta\left(\mathbf{A}^{H} \mathbf{b}\right)_{1}^{(k=2)}\right)^{2}\right) \\
& =\sigma_{\phi}^{2} \sum_{i=1}^{N}\left(x_{i} \sin 2 \phi_{i}-y_{i} \cos 2 \phi_{i}\right)^{2} \\
& +3 \sigma_{\phi}^{4} \sum_{i=1}^{N}\left(x_{i} \cos 2 \phi_{i}-y_{i} \sin 2 \phi_{i}\right)^{2} \\
& +\sigma_{\phi}^{4} \sum_{i=1(i \neq j)}^{N} \sum_{j=1}^{N}\left(x_{i} \cos 2 \phi_{i}-y_{i} \sin 2 \phi_{i}\right) \\
& \cdot\left(x_{j} \cos 2 \phi_{j}-y_{j} \sin 2 \phi_{j}\right) \text {, }
\end{aligned}
$$




$$
\begin{aligned}
& E\left(\left(\delta\left(\mathbf{A}^{H} \mathbf{b}\right)_{2}^{(k=2)}\right)^{2}\right) \\
& =\sigma_{\phi}^{2} \sum_{i=1}^{N}\left(-x_{i} \cos 2 \phi_{i}-y_{i} \sin 2 \phi_{i}\right)^{2} \\
& +3 \sigma_{\phi}^{4} \sum_{i=1}^{N}\left(x_{i} \sin 2 \phi_{i}-y_{i} \cos 2 \phi_{i}\right)^{2} \\
& +\sigma_{\phi}^{4} \sum_{i=1(i \neq j)}^{N} \sum_{j=1}^{N}\left(x_{i} \sin 2 \phi_{i}-y_{i} \cos 2 \phi_{i}\right) \\
& \cdot\left(x_{j} \sin 2 \phi_{j}-y_{j} \cos 2 \phi_{j}\right) \text {, } \\
& E\left(\left(\delta\left(\mathbf{A}^{H} \mathbf{A}\right)_{11}^{(k=2)}\right)\left(\delta\left(\mathbf{A}^{H} \mathbf{A}\right)_{12}^{(k=2)}\right)\right) \\
& =\sigma_{\phi}^{2} \sum_{i=1}^{N} \sin 2 \phi_{i}\left(-\cos 2 \phi_{i}\right)+3 \sigma_{\phi}^{4} \sum_{i=1}^{N} \cos 2 \phi_{i} \sin 2 \phi_{i} \\
& +\sigma_{\phi}^{4} \sum_{i=1(i \neq j)}^{N} \sum_{j=1}^{N} \cos 2 \phi_{i} \sin 2 \phi_{j} \\
& E\left(\left(\delta\left(\mathbf{A}^{H} \mathbf{A}\right)_{11}^{(k=2)}\right)\left(\delta\left(\mathbf{A}^{H} \mathbf{A}\right)_{22}^{(k=2)}\right)\right) \\
& =\sigma_{\phi}^{2} \sum_{i=1}^{N} \sin 2 \phi_{i}\left(-\sin 2 \phi_{i}\right)+3 \sigma_{\phi}^{4} \sum_{i=1}^{N} \cos 2 \phi_{i}\left(-\cos 2 \phi_{i}\right) \\
& +\sigma_{\phi}^{4} \sum_{i=1(i \neq j)}^{N} \sum_{j=1}^{N} \cos 2 \phi_{i}\left(-\cos 2 \phi_{j}\right) \\
& E\left(\left(\delta\left(\mathbf{A}^{H} \mathbf{A}\right)_{12}^{(k=2)}\right)\left(\delta\left(\mathbf{A}^{H} \mathbf{A}\right)_{22}^{(k=2)}\right)\right) \\
& =\sigma_{\phi}^{2} \sum_{i=1}^{N}\left(-\cos 2 \phi_{i}\right)\left(-\sin 2 \phi_{i}\right)+3 \sigma_{\phi}^{4} \sum_{i=1}^{N} \sin 2 \phi_{i}\left(-\cos 2 \phi_{i}\right) \\
& +\sigma_{\phi}^{4} \sum_{i=1(i \neq j)}^{N} \sum_{j=1}^{N} \sin 2 \phi_{i}\left(-\cos 2 \phi_{j}\right) \\
& E\left(\left(\delta\left(\mathbf{A}^{H} \mathbf{A}\right)_{11}^{(k=2)}\right)\left(\delta\left(\mathbf{A}^{H} \mathbf{b}\right)_{1}^{(k=2)}\right)\right) \\
& =\sigma_{\phi}^{2} \sum_{i=1}^{N}\left(\sin 2 \phi_{i}\right)\left(x_{i} \sin 2 \phi_{i}-y_{i} \cos 2 \phi_{i}\right) \\
& +3 \sigma_{\phi}^{4} \sum_{i=1}^{N}\left(\cos 2 \phi_{i}\right)\left(x_{i} \cos 2 \phi_{i}-y_{i} \sin 2 \phi_{i}\right) \\
& +\sigma_{\phi}^{4} \sum_{i=1(i \neq j)}^{N} \sum_{j=1}^{N}\left(\cos 2 \phi_{i}\right)\left(x_{i} \cos 2 \phi_{i}-y_{i} \sin 2 \phi_{i}\right) \text {, } \\
& E\left(\left(\delta\left(\mathbf{A}^{H} \mathbf{A}\right)_{12}^{(k=2)}\right)\left(\delta\left(\mathbf{A}^{H} \mathbf{b}\right)_{1}^{(k=2)}\right)\right) \\
& =\sigma_{\phi}^{2} \sum_{i=1}^{N}\left(-\cos 2 \phi_{i}\right)\left(x_{i} \sin 2 \phi_{i}-y_{i} \cos 2 \phi_{i}\right) \\
& +3 \sigma_{\phi}^{4} \sum_{i=1}^{N}\left(\sin 2 \phi_{i}\right)\left(x_{i} \cos 2 \phi_{i}-y_{i} \sin 2 \phi_{i}\right) \\
& +\sigma_{\phi}^{4} \sum_{i=1(i \neq j)}^{N} \sum_{j=1}^{N}\left(\sin 2 \phi_{i}\right)\left(x_{i} \cos 2 \phi_{i}-y_{i} \sin 2 \phi_{i}\right) \text {, } \\
& E\left(\left(\delta\left(\mathbf{A}^{H} \mathbf{A}\right)_{22}^{(k=2)}\right)\left(\delta\left(\mathbf{A}^{H} \mathbf{b}\right)_{1}^{(k=2)}\right)\right) \\
& =\sigma_{\phi}^{2} \sum_{i=1}^{N}\left(-\sin \phi_{i}\right)\left(x_{i} \sin 2 \phi_{i}-y_{i} \cos 2 \phi_{i}\right) \\
& -3 \sigma_{\phi}^{4} \sum_{i=1}^{N}\left(\cos 2 \phi_{i}\right)\left(x_{i} \cos 2 \phi_{i}-y_{i} \sin 2 \phi_{i}\right) \\
& -\sigma_{\phi}^{4} \sum_{i=1(i \neq j)}^{N} \sum_{j=1}^{N} \cos 2 \phi_{i}\left(x_{i} \cos 2 \phi_{i}-y_{i} \sin 2 \phi_{i}\right) \\
& E\left(\left(\delta\left(\mathbf{A}^{H} \mathbf{A}\right)_{1}^{(k=2)}\right)\left(\delta\left(\mathbf{A}^{H} \mathbf{b}\right)_{2}^{(k=2)}\right)\right) \\
& =\sigma_{\phi}^{2} \sum_{i=1}^{N}\left(x_{i} \sin 2 \phi_{i}-y_{i} \cos 2 \phi_{i}\right)\left(-x_{i} \cos 2 \phi_{i}-y_{i} \sin 2 \phi_{i}\right) \\
& +3 \sigma_{\phi}^{4} \sum_{i=1}^{N}\left(x_{i} \cos 2 \phi_{i}-y_{i} \sin 2 \phi_{i}\right)\left(x_{i} \sin 2 \phi_{i}-y_{i} \cos 2 \phi_{i}\right) \\
& +\sigma_{\phi}^{4} \sum_{i=1(i \neq j)}^{N} \sum_{j=1}^{N}\left(x_{i} \cos 2 \phi_{i}-y_{i} \sin 2 \phi_{i}\right) \\
& \cdot\left(x_{i} \sin 2 \phi_{i}-y_{i} \cos 2 \phi_{i}\right) \text {, } \\
& E\left(\left(\delta\left(\mathbf{A}^{H} \mathbf{A}\right)_{11}^{(k=2)}\right)\left(\delta\left(\mathbf{A}^{H} \mathbf{b}\right)_{2}^{(k=2)}\right)\right) \\
& =\sigma_{\phi}^{2} \sum_{i=1}^{N} \sin 2 \phi_{i}\left(-x_{i} \cos 2 \phi_{i}-y_{i} \sin 2 \phi_{i}\right) \\
& +3 \sigma_{\phi}^{4} \sum_{i=1}^{N} \cos 2 \phi_{i}\left(x_{i} \sin 2 \phi_{i}-y_{i} \cos 2 \phi_{i}\right) \\
& +\sigma_{\phi}^{4} \sum_{i=1(i \neq j)}^{N} \sum_{j=1}^{N} \cos 2 \phi_{i}\left(x_{i} \sin 2 \phi_{i}-y_{i} \cos 2 \phi_{i}\right), \\
& E\left(\left(\delta\left(\mathbf{A}^{H} \mathbf{A}\right)_{12}^{(k=2)}\right)\left(\delta\left(\mathbf{A}^{H} \mathbf{b}\right)_{2}^{(k=2)}\right)\right) \\
& =\sigma_{\phi}^{2} \sum_{i=1}^{N}\left(-\cos 2 \phi_{i}\right)\left(-x_{i} \cos 2 \phi_{i}-y_{i} \sin 2 \phi_{i}\right) \\
& +3 \sigma_{\phi}^{4} \sum_{i=1}^{N} \sin \phi_{i}\left(x_{i} \sin 2 \phi_{i}-y_{i} \cos 2 \phi_{i}\right) \\
& +\sigma_{\phi}^{4} \sum_{i=1(i \neq j)}^{N} \sum_{j=1}^{N} \sin 2 \phi_{i}\left(x_{i} \sin 2 \phi_{i}-y_{i} \cos 2 \phi_{i}\right),
\end{aligned}
$$




$$
\begin{aligned}
E(( & \left.\left.\delta\left(\mathbf{A}^{H} \mathbf{A}\right)_{22}^{(k=2)}\right)\left(\delta\left(\mathbf{A}^{H} \mathbf{b}\right)_{2}^{(k=2)}\right)\right) \\
= & \sigma_{\phi}^{2} \sum_{i=1}^{N}\left(-\sin 2 \phi_{i}\right)\left(-x_{i} \cos 2 \phi_{i}-y_{i} \sin 2 \phi_{i}\right) \\
& +3 \sigma_{\phi}^{4} \sum_{i=1}^{N}\left(-\cos 2 \phi_{i}\right)\left(x_{i} \sin 2 \phi_{i}-y_{i} \cos 2 \phi_{i}\right) \\
& +\sigma_{\phi}^{4} \sum_{i=1(i \neq j)}^{N} \sum_{j=1}^{N}\left(-\cos 2 \phi_{i}\right)\left(x_{i} \sin 2 \phi_{i}-y_{i} \cos 2 \phi_{i}\right),
\end{aligned}
$$

F. Mean of Entries of $\delta\left(\mathbf{A}^{\prime H} \mathbf{A}^{\prime}\right)$ and $\delta\left(\mathbf{A}^{\prime}{ }^{H} \mathbf{b}^{\prime}\right)$ Based on the Third-Order Taylor Series

$$
\begin{aligned}
& E(\left.\left(\delta\left(\mathbf{A}^{H} \mathbf{A}\right)_{11}^{(k=3)}\right)^{2}\right) \\
&= \sigma_{\phi}^{2} \sum_{i=1}^{N} \sin ^{2} 2 \phi_{i}+3 \sigma_{\phi}^{4} \sum_{i=1}^{N} \cos ^{2} 2 \phi_{i} \\
&+\sigma_{\phi}^{4} \sum_{i=1(i \neq j)}^{N} \sum_{j=1}^{N} \cos 2 \phi_{i} \cos 2 \phi_{j} \\
&+6 \sigma_{\phi}^{4} \sum_{i=1}^{N}\left(-\frac{2}{3} \sin 2 \phi_{i}\right)\left(\sin 2 \phi_{i}\right) \\
&+15 \sigma_{\phi}^{6} \sum_{i=1}^{N}\left(-\frac{2}{3} \sin 2 \phi_{i}\right)\left(-\frac{2}{3} \sin 2 \phi\right), \\
& E\left(\left(\delta\left(\mathbf{A}^{H} \mathbf{A}\right)_{12}^{(k=3)}\right)^{2}\right) \\
&=\sigma_{\phi}^{2} \sum_{i=1}^{N} \cos 2 \phi_{i}+3 \sigma_{\phi}^{4} \sum_{i=1}^{N} \sin ^{2} 2 \phi_{i} \\
&+\sigma_{\phi}^{4} \sum_{i=1(i \neq j)}^{N} \sum_{j=1}^{N} \sin 2 \phi_{i} \sin 2 \phi_{j}+6 \sigma_{\phi}^{4} \sum_{i=1}^{N} \\
&-\left(\cos 2 \phi_{i}\right)\left(\frac{2}{3} \cos 2 \phi_{i}\right)+15 \sigma_{\phi}^{6} \sum_{i=1}^{N}\left(\frac{2}{3} \cos 2 \phi_{i}\right) \\
& \quad\left(\frac{2}{3} \cos 2 \phi_{i}\right),
\end{aligned}
$$

$$
\begin{aligned}
E(( & \left.\left.\delta\left(\mathbf{A}^{H} \mathbf{A}\right)_{22}^{(k=3)}\right)^{2}\right) \\
= & \sigma_{\phi}^{2} \sum_{i=1}^{N} \sin ^{2} 2 \phi_{i}+3 \sigma_{\phi}^{4} \sum_{i=1}^{N} \cos ^{2} 2 \phi_{i} \\
& +\sigma_{\phi}^{4} \sum_{i=1(i \neq j)}^{N} \sum_{j=1}^{N} \cos 2 \phi_{i} \cos 2 \phi_{j}+6 \sigma_{\phi}^{4} \sum_{i=1}^{N} \\
& -\left(\sin 2 \phi_{i}\right)\left(\frac{2}{3} \sin 2 \phi_{i}\right)+15 \sigma_{\phi}^{6} \sum_{i=1}^{N}\left(\frac{2}{3} \sin 2 \phi_{i}\right) \\
& \cdot\left(\frac{2}{3} \sin 2 \phi_{i}\right),
\end{aligned}
$$

$$
\begin{aligned}
E(( & \left.\left.\delta\left(\mathbf{A}^{H} \mathbf{b}\right)_{2}^{(k=3)}\right)^{2}\right) \\
= & \sigma_{\phi}^{2} \sum_{i=1}^{N}\left(-x_{i} \cos 2 \phi_{i}-y_{i} \sin 2 \phi_{i}\right)^{2} \\
& +3 \sigma_{\phi}^{4} \sum_{i=1}^{N}\left(x_{i} \sin 2 \phi_{i}-y_{i} \cos 2 \phi_{i}\right)^{2} \\
& +\sigma_{\phi}^{4} \sum_{i=1(i \neq j)}^{N} \sum_{j=1}^{N}\left(x_{i} \sin 2 \phi_{i}-y_{i} \cos 2 \phi_{i}\right) \\
& +\left(x_{j} \sin 2 \phi_{j}-y_{j} \cos 2 \phi_{j}\right) \\
& +6 \sigma_{\phi}^{4} \sum_{i=1}^{N}\left(x_{i}\left(\frac{2}{3} \cos 2 \phi_{i}\right)+y_{i}\left(\frac{2}{3} \sin 2 \phi_{i}\right)\right) \\
& +\left(-x_{i} \cos 2 \phi_{i}-y_{i} \sin 2 \phi_{i}\right) \\
& +15 \sigma_{\phi}^{6} \sum_{i=1}^{N}\left(x_{i}\left(\frac{2}{3} \cos 2 \phi_{i}\right)+y_{i}\left(\frac{2}{3} \sin 2 \phi_{i}\right)\right)^{2},
\end{aligned}
$$

$$
\begin{aligned}
E( & \left.\left(\delta\left(\mathbf{A}^{H} \mathbf{A}\right)_{11}^{(k=3)}\right)\left(\delta\left(\mathbf{A}^{H} \mathbf{A}\right)_{12}^{(k=3)}\right)\right) \\
= & \sigma_{\phi}^{2} \sum_{i=1}^{N} \sin 2 \phi_{i}\left(-\cos 2 \phi_{i}\right)+3 \sigma_{\phi}^{4} \sum_{i=1}^{N} \cos 2 \phi_{i} \sin 2 \phi_{i} \\
& +\sigma_{\phi}^{4} \sum_{i=1(i \neq j)}^{N} \sum_{j=1}^{N} \cos 2 \phi_{i} \sin 2 \phi_{j}+3 \sigma_{\phi}^{4} \sum_{i=1}^{N}\left(\sin 2 \phi_{i}\right) \\
& \cdot\left(\frac{2}{3} \cos 2 \phi_{i}\right)+3 \sigma_{\phi}^{4} \sum_{i=1}^{N}\left(-\frac{2}{3} \sin 2 \phi_{i}\right)\left(-\cos 2 \phi_{i}\right) \\
& +15 \sigma_{\phi}^{6} \sum_{i=1}^{N}\left(-\frac{2}{3} \sin 2 \phi_{i}\right)\left(\frac{2}{3} \cos 2 \phi_{i}\right),
\end{aligned}
$$

(F.3) 


$$
\begin{aligned}
E(( & \left.\left.\left(\mathbf{A}^{H} \mathbf{A}\right)_{11}^{(k=3)}\right)\left(\delta\left(\mathbf{A}^{H} \mathbf{A}\right)_{22}^{(k=3)}\right)\right) \\
= & \sigma_{\phi}^{2} \sum_{i=1}^{N} \sin 2 \phi_{i}\left(-\sin 2 \phi_{i}\right)+3 \sigma_{\phi}^{4} \sum_{i=1}^{N} \cos 2 \phi_{i}\left(-\cos 2 \phi_{i}\right) \\
& +\sigma_{\phi}^{4} \sum_{i=1(i \neq j)}^{N} \sum_{j=1}^{N} \cos 2 \phi_{i}\left(-\cos 2 \phi_{j}\right)+3 \sigma_{\phi}^{4} \sum_{i=1}^{N}\left(\sin 2 \phi_{i}\right) \\
& \cdot\left(\frac{2}{3} \sin 2 \phi_{i}\right)+3 \sigma_{\phi}^{4} \sum_{i=1}^{N}\left(-\sin 2 \phi_{i}\right)\left(-\frac{2}{3} \sin 2 \phi_{i}\right) \\
& +15 \sigma_{\phi}^{6} \sum_{i=1}^{N}\left(-\frac{2}{3} \sin 2 \phi_{i}\right)\left(\frac{2}{3} \sin 2 \phi_{i}\right)
\end{aligned}
$$

$$
\begin{aligned}
E(( & \left.\left.\delta\left(\mathbf{A}^{H} \mathbf{A}\right)_{12}^{(k=3)}\right)\left(\delta\left(\mathbf{A}^{H} \mathbf{A}\right)_{22}^{(k=3)}\right)\right) \\
= & \sigma_{\phi}^{2} \sum_{i=1}^{N}\left(-\cos 2 \phi_{i}\right)\left(-\sin 2 \phi_{i}\right)+3 \sigma_{\phi}^{4} \sum_{i=1}^{N} \sin 2 \phi_{i}\left(-\cos 2 \phi_{i}\right) \\
& +\sigma_{\phi}^{4} \sum_{i=1(i \neq j)}^{N} \sum_{j=1}^{N} \sin 2 \phi_{i}\left(-\cos 2 \phi_{j}\right)+3 \sigma_{\phi}^{4} \sum_{i=1}^{N}\left(-\cos 2 \phi_{i}\right) \\
& \cdot\left(\frac{2}{3} \sin 2 \phi_{i}\right)+3 \sigma_{\phi}^{4} \sum_{i=1}^{N}\left(-\sin 2 \phi_{i}\right)\left(\frac{2}{3} \cos 2 \phi_{i}\right) \\
& +15 \sigma_{\phi}^{6} \sum_{i=1}^{N}\left(\frac{2}{3} \cos 2 \phi_{i}\right)\left(\frac{2}{3} \sin 2 \phi_{i}\right)
\end{aligned}
$$

$$
\begin{aligned}
E(( & \left.\left.\delta\left(\mathbf{A}^{H} \mathbf{A}\right)_{11}^{(k=3)}\right)\left(\delta\left(\mathbf{A}^{H} \mathbf{b}\right)_{1}^{(k=3)}\right)\right) \\
= & \sigma_{\phi}^{2} \sum_{i=1}^{N}\left(\sin 2 \phi_{i}\right)\left(x_{i} \sin 2 \phi_{i}-y_{i} \cos 2 \phi_{i}\right) \\
& +3 \sigma_{\phi}^{4} \sum_{i=1}^{N}\left(\cos 2 \phi_{i}\right)\left(x_{i} \cos 2 \phi_{i}-y_{i} \sin 2 \phi_{i}\right) \\
& +\sigma_{\phi}^{4} \sum_{i=1(i \neq j)}^{N} \sum_{j=1}^{N}\left(\cos 2 \phi_{i}\right)\left(x_{i} \cos 2 \phi_{i}-y_{i} \sin 2 \phi_{i}\right) \\
& +3 \sigma_{\phi}^{4} \sum_{i=1}^{N}\left(\sin 2 \phi_{i}\right)\left(x_{i}\left(-\frac{2}{3} \sin 2 \phi_{i}\right)+y_{i}\left(\frac{2}{3} \cos 2 \phi_{i}\right)\right) \\
& -3 \sigma_{\phi}^{4} \sum_{i=1}^{N}\left(\frac{2}{3} \sin 2 \phi_{i}\right)\left(x_{i} \sin 2 \phi_{i}-y_{i} \cos 2 \phi_{i}\right) \\
& +15 \sigma_{\phi}^{6} \sum_{i=1}^{N}\left(-\frac{2}{3} \sin 2 \phi_{i}\right)\left(x_{i}\left(-\frac{2}{3} \sin 2 \phi_{i}\right)\right. \\
& \left.+y_{i}\left(\frac{2}{3} \cos 2 \phi_{i}\right)\right),
\end{aligned}
$$

$$
\begin{aligned}
E(( & \left.\left.\delta\left(\mathbf{A}^{H} \mathbf{A}\right)_{12}^{(k=3)}\right)\left(\delta\left(\mathbf{A}^{H} \mathbf{b}\right)_{1}^{(k=3)}\right)\right) \\
= & \sigma_{\phi}^{2} \sum_{i=1}^{N}\left(-\cos 2 \phi_{i}\right)\left(x_{i} \sin 2 \phi_{i}-y_{i} \cos 2 \phi_{i}\right) \\
& +3 \sigma_{\phi}^{4} \sum_{i=1}^{N}\left(\sin 2 \phi_{i}\right)\left(x_{i} \cos 2 \phi_{i}-y_{i} \sin 2 \phi_{i}\right) \\
& +\sigma_{\phi}^{4} \sum_{i=1(i \neq j)}^{N} \sum_{j=1}^{N}\left(\sin 2 \phi_{i}\right)\left(x_{i} \cos 2 \phi_{i}-y_{i} \sin 2 \phi_{i}\right) \\
& +3 \sigma_{\phi}^{4} \sum_{i=1}^{N}\left(-\cos 2 \phi_{i}\right)\left(x_{i}\left(-\frac{2}{3} \sin 2 \phi_{i}\right)\right. \\
& \left.+y_{i}\left(\frac{2}{3} \cos 2 \phi_{i}\right)\right)+3 \sigma_{\phi}^{4} \sum_{i=1}^{N}\left(\frac{2}{3} \cos 2 \phi_{i}\right) \\
& +\left(x_{i} \sin 2 \phi_{i}-y_{i} \cos 2 \phi_{i}\right)+15 \sigma_{\phi}^{6} \sum_{i=1}^{N}\left(\frac{2}{3} \cos 2 \phi_{i}\right) \\
& +\left(x_{i}\left(-\frac{2}{3} \sin 2 \phi_{i}\right)+y_{i}\left(\frac{2}{3} \cos 2 \phi_{i}\right)\right),
\end{aligned}
$$$$
E\left(\left(\delta\left(\mathbf{A}^{H} \mathbf{A}\right)_{22}^{(k=3)}\right)\left(\delta\left(\mathbf{A}^{H} \mathbf{b}\right)_{1}^{(k=3)}\right)\right)
$$$$
=\sigma_{\phi}^{2} \sum_{i=1}^{N}\left(-\sin \phi_{i}\right)\left(x_{i} \sin 2 \phi_{i}-y_{i} \cos 2 \phi_{i}\right)
$$$$
-3 \sigma_{\phi}^{4} \sum_{i=1}^{N}\left(\cos 2 \phi_{i}\right)\left(x_{i} \cos 2 \phi_{i}-y_{i} \sin 2 \phi_{i}\right)
$$$$
-\sigma_{\phi}^{4} \sum_{i=1(i \neq j)}^{N} \sum_{j=1}^{N} \cos 2 \phi_{i}\left(x_{i} \cos 2 \phi_{i}-y_{i} \sin 2 \phi_{i}\right)
$$$$
+3 \sigma_{\phi}^{4} \sum_{i=1}^{N}\left(-\sin 2 \phi_{i}\right)\left(x_{i}\left(-\frac{2}{3} \sin 2 \phi_{i}\right)\right.
$$$$
\left.+y_{i}\left(\frac{2}{3} \cos 2 \phi_{i}\right)\right)+3 \sigma_{\phi}^{4} \sum_{i=1}^{N}\left(\frac{2}{3} \sin 2 \phi_{i}\right)
$$$$
\cdot\left(x_{i} \sin 2 \phi_{i}-y_{i} \cos 2 \phi_{i}\right)+15 \sigma_{\phi}^{6} \sum_{i=1}^{N}\left(\frac{2}{3} \sin 2 \phi_{i}\right)
$$$$
\cdot\left(x_{i}\left(-\frac{2}{3} \sin 2 \phi_{i}\right)+y_{i}\left(\frac{2}{3} \cos 2 \phi_{i}\right)\right) \text {, }
$$$$
E\left(\left(\delta\left(\mathbf{A}^{H} \mathbf{A}\right)_{1}^{(k=3)}\right)\left(\delta\left(\mathbf{A}^{H} \mathbf{b}\right)_{2}^{(k=3)}\right)\right)
$$$$
=\sigma_{\phi}^{2} \sum_{i=1}^{N}\left(x_{i} \sin 2 \phi_{i}-y_{i} \cos 2 \phi_{i}\right)\left(-x_{i} \cos 2 \phi_{i}-y_{i} \sin 2 \phi_{i}\right)
$$$$
+3 \sigma_{\phi}^{4} \sum_{i=1}^{N}\left(x_{i} \cos 2 \phi_{i}-y_{i} \sin 2 \phi_{i}\right)\left(x_{i} \sin 2 \phi_{i}-y_{i} \cos 2 \phi_{i}\right)
$$$$
+\sigma_{\phi}^{4} \sum_{i=1(i \neq j)}^{N} \sum_{j=1}^{N}\left(x_{i} \cos 2 \phi_{i}-y_{i} \sin 2 \phi_{i}\right)\left(x_{i} \sin 2 \phi_{i}\right.
$$$$
\left.-y_{i} \cos 2 \phi_{i}\right)+3 \sigma_{\phi}^{4} \sum_{i=1}^{N}\left(x_{i} \sin 2 \phi_{i}-y_{i} \cos 2 \phi_{i}\right)
$$$$
\cdot\left(x_{i}\left(\frac{2}{3} \cos 2 \phi_{i}\right)+y_{i}\left(\frac{2}{3} \sin 2 \phi_{i}\right)\right)
$$$$
+3 \sigma_{\phi}^{4} \sum_{i=1}^{N}\left(-x_{i} \cos 2 \phi_{i}-y_{i} \sin 2 \phi_{i}\right)\left(x_{i}\left(-\frac{2}{3} \sin 2 \phi_{i}\right)\right.
$$$$
\left.+y_{i}\left(\frac{2}{3} \cos 2 \phi_{i}\right)\right)+15 \sigma_{\phi}^{6} \sum_{i=1}^{N}\left(x_{i}\left(-\frac{2}{3} \sin 2 \phi_{i}\right)\right.
$$$$
\left.+y_{i}\left(\frac{2}{3} \cos 2 \phi_{i}\right)\right)\left(x_{i}\left(\frac{2}{3} \cos 2 \phi_{i}\right)+y_{i}\left(\frac{2}{3} \sin 2 \phi_{i}\right)\right) \text {, }
$$ 


$$
\begin{aligned}
E(( & \left.\left.\delta\left(\mathbf{A}^{H} \mathbf{A}\right)_{11}^{(k=3)}\right)\left(\delta\left(\mathbf{A}^{H} \mathbf{b}\right)_{2}^{(k=3)}\right)\right) \\
= & \sigma_{\phi}^{2} \sum_{i=1}^{N} \sin 2 \phi_{i}\left(-x_{i} \cos 2 \phi_{i}-y_{i} \sin 2 \phi_{i}\right) \\
& +3 \sigma_{\phi}^{4} \sum_{i=1}^{N} \cos 2 \phi_{i}\left(x_{i} \sin 2 \phi_{i}-y_{i} \cos 2 \phi_{i}\right) \\
& +\sigma_{\phi}^{4} \sum_{i=1(i \neq j)}^{N} \sum_{j=1}^{N} \cos 2 \phi_{i}\left(x_{i} \sin 2 \phi_{i}-y_{i} \cos 2 \phi_{i}\right) \\
& +3 \sigma_{\phi}^{4} \sum_{i=1}^{N}\left(\sin 2 \phi_{i} x_{i}\left(\frac{2}{3} \cos 2 \phi_{i}\right)+y_{i}\left(\frac{2}{3} \sin 2 \phi_{i}\right)\right) \\
& +3 \sigma_{\phi}^{4} \sum_{i=1}^{N}\left(-\frac{2}{3} \sin 2 \phi_{i}\right)\left(-x_{i} \cos 2 \phi_{i}-y_{i} \sin 2 \phi_{i}\right) \\
& +15 \sigma_{\phi}^{6} \sum_{i=1}^{N}\left(-\frac{2}{3} \sin 2 \phi_{i}\right)\left(x_{i}\left(\frac{2}{3} \cos 2 \phi_{i}\right)\right. \\
& \left.+y_{i}\left(\frac{2}{3} \sin 2 \phi_{i}\right)\right)
\end{aligned}
$$

$$
\begin{aligned}
E(( & \left.\left.\delta\left(\mathbf{A}^{H} \mathbf{A}\right)_{12}^{(k=3)}\right)\left(\delta\left(\mathbf{A}^{H} \mathbf{b}\right)_{2}^{(k=3)}\right)\right) \\
= & \sigma_{\phi}^{2} \sum_{i=1}^{N}\left(-\cos 2 \phi_{i}\right)\left(-x_{i} \cos 2 \phi_{i}-y_{i} \sin 2 \phi_{i}\right) \\
& +3 \sigma_{\phi}^{4} \sum_{i=1}^{N} \sin \phi_{i}\left(x_{i} \sin 2 \phi_{i}-y_{i} \cos 2 \phi_{i}\right) \\
& +\sigma_{\phi}^{4} \sum_{i=1(i \neq j)}^{N} \sum_{j=1}^{N} \sin 2 \phi_{i}\left(x_{i} \sin 2 \phi_{i}-y_{i} \cos 2 \phi_{i}\right) \\
& +3 \sigma_{\phi}^{4} \sum_{i=1}^{N}\left(-\cos 2 \phi_{i}\right)\left(x_{i}\left(\frac{2}{3} \cos 2 \phi_{i}\right)+y_{i}\left(\frac{2}{3} \sin 2 \phi_{i}\right)\right) \\
& +3 \sigma_{\phi}^{4} \sum_{i=1}^{N} \cos 2 \phi_{i}\left(-x_{i} \cos 2 \phi_{i}-y_{i} \sin 2 \phi_{i}\right) \\
& +15 \sigma_{\phi}^{6} \sum_{i=1}^{N}\left(\frac{2}{3} \cos 2 \phi_{i}\right)\left(x_{i}\left(\frac{2}{3} \cos 2 \phi_{i}\right)\right. \\
& \left.+y_{i}\left(\frac{2}{3} \sin 2 \phi_{i}\right)\right),
\end{aligned}
$$

$$
\begin{aligned}
E( & \left.\left(\delta\left(\mathbf{A}^{H} \mathbf{A}\right)_{22}^{(k=3)}\right)\left(\delta\left(\mathbf{A}^{H} \mathbf{b}\right)_{2}^{(k=3)}\right)\right) \\
= & \sigma_{\phi}^{2} \sum_{i=1}^{N}\left(-\sin 2 \phi_{i}\right)\left(-x_{i} \cos 2 \phi_{i}-y_{i} \sin 2 \phi_{i}\right) \\
& +3 \sigma_{\phi}^{4} \sum_{i=1}^{N}\left(-\cos 2 \phi_{i}\right)\left(x_{i} \sin 2 \phi_{i}-y_{i} \cos 2 \phi_{i}\right) \\
& +\sigma_{\phi}^{4} \sum_{i=1(i \neq j)}^{N} \sum_{j=1}^{N}\left(-\cos 2 \phi_{i}\right)\left(x_{i} \sin 2 \phi_{i}-y_{i} \cos 2 \phi_{i}\right) \\
& +3 \sigma_{\phi}^{4} \sum_{i=1}^{N}\left(-\sin 2 \phi_{i}\right)\left(x_{i}\left(\frac{2}{3} \cos 2 \phi_{i}\right)+y_{i}\left(\frac{2}{3} \sin 2 \phi_{i}\right)\right) \\
& +3 \sigma_{\phi}^{4} \sum_{i=1}^{N}\left(\frac{2}{3} \sin 2 \phi_{i}\right)\left(-x_{i} \cos 2 \phi_{i}-y_{i} \sin 2 \phi_{i}\right) \\
& +15 \sigma_{\phi}^{6} \sum_{i=1}^{N}\left(\frac{2}{3} \sin 2 \phi_{i}\right)\left(x_{i}\left(\frac{2}{3} \cos 2 \phi_{i}\right)\right. \\
& \left.+y_{i}\left(\frac{2}{3} \sin 2 \phi_{i}\right)\right) .
\end{aligned}
$$

\section{Data Availability}

The data used to support the findings of this study are available from the corresponding author upon request.

\section{Conflicts of Interest}

The authors declare that they have no conflicts of interest.

\section{Acknowledgments}

This research was supported by Basic Science Research Program through the National Research Foundation of Korea (NRF) funded by the Ministry of Education (2018R1D1A1B07048294).

\section{References}

[1] A. Pages-Zamora, J. Vidal, and D. H. Brooks, "Closed-form solution for positioning based on angle of arrival measurements," in The 13th IEEE International Symposium on Personal, Indoor and Mobile Radio Communications, pp. 1522-1526, Pavilhao Altantico, Lisboa, Portugal, Portugal, 2002.

[2] G. Sun, X. Xu, and K. Yang, "Angle-of-Arrival based Constrained Total Least-Squares Location Algorithm," in Proceedings of 2012 National Conference on Information Technology and Computer Science, 2012.

[3] K. Doğançay and H. Hmam, "Optimal angular sensor separation for AOA localization," Signal Processing, vol. 88, no. 5, pp. 1248-1260, 2008.

[4] J. Xu, M. Ma, and C. L. Law, "AOA Cooperative Position Localization," in IEEE GLOBECOM 2008 - 2008 IEEE Global Telecommunications Conference, pp. 1-5, New Orleans, LO, USA, 2008.

[5] H. Tian, S. Wang, and H. Xie, "Localization using cooperative AOA approach," in 2007 International Conference on Wireless Communications, Networking and Mobile Computing, pp. 2416-2419, Shanghai, China, 2007.

[6] Y. Wang and K. C. Ho, "An Asymptotically Efficient Estimator in Closed-Form for 3-D AOA Localization Using a Sensor Network," IEEE Transactions on Wireless Communications, vol. 14, no. 12, pp. 6524-6535, 2015.

[7] P. Kułakowski, J. Vales-Alonso, E. Egea-López, W. Ludwin, and J. García-Haro, "Angle-of-arrival localization based on antenna arrays for wireless sensor networks," Computers and Electrical Engineering, vol. 36, no. 6, pp. 1181-1186, 2010.

[8] L. Rui and K. C. Ho, "Bias analysis of source localization using the maximum likelihood estimator," in 2012 IEEE International Conference on Acoustics, Speech and Signal Processing (ICASSP), pp. 2605-2608, Kyoto, Japan, 2012.

[9] A. N. Bishop, B. Fidan, B. D. O. Anderson, K. Dogancay, and P. N. Pathirana, "Optimality analysis of sensor-target geometries in passive localization: part 1 - bearing-only localization," in 2007 3rd International Conference on Intelligent Sensors, Sensor Networks and Information, pp. 7-12, Melbourne, Qld., Australia, 2007.

[10] R. M. Brown, "Emitter location using bearing measurement from a moving platform," Naval Research Laboratory, Washington, D.C., 1981.

[11] J. L. Poirot and M. S. Smith, "Moving emitter classification," IEEE Transactions on Aerospace and Electronic Systems, vol. AES-12, no. 2, pp. 255-269, 1976. 
[12] J. L. Poirot and G. V. McWilliams, "Application of linear statistical models to radar location techniques," IEEE Transactions on Aerospace and Electronic Systems, vol. AES-10, no. 6, pp. 830-834, 1974.

[13] K. Spingarn, "Passive position location estimation using the extended Kalman filter," IEEE Transactions on Aerospace and Electronic Systems, vol. AES-23, no. 4, pp. 558-567, 1987.

[14] M. Gavish and A. J. Weiss, "Performance analysis of bearingonly target location algorithms," IEEE Transactions on Aerospace and Electronic Systems, vol. 28, no. 3, pp. 817-828, 1992.

[15] G. Golub and C. Van Loan, "An Analysis of the Total Least Squares Problem," SIAM Journal on Numerical Analysis, vol. 17, no. 6, pp. 883-893, 1980.

[16] D. J. An, C.-M. Moon, and J.-H. Lee, "Derivation of an explicit expression of an approximate location estimate in AOA-based localization," in 2017 7th IEEE International Symposium on Microwave, Antenna, Propagation, and EMC Technologies (MAPE), pp. 552-555, Xi'an, China, 2017.

[17] D. Torrieri, "Statistical theory of passive location systems," IEEE Transactions on Aerospace and Electronic Systems, vol. AES-20, no. 2, pp. 183-198, 1984.

[18] M. Kaminski, "On iterative scheme in determination of the probabilistic moments of the structural response in the stochastic perturbation-based finite element method," International Journal for Numerical Methods in Engineering, vol. 104, no. 11, pp. 1038-1060, 2015.

[19] M. Kaminski, The Stochastic Perturbation Method for Computational Mechanics, Wiley, The Atrium, Southern Gate, Chichester, West Sussex, PO19 8SQ, United Kingdom, 2013. 